\title{
TITLE:
}

\section{Bifurcations and instabilities in sliding Couette flow}

\author{
$\operatorname{AUTHOR}(\mathrm{S})$ :
}

Deguchi, K.; Nagata, M.

\section{CITATION:}

Deguchi, K.... [et al]. Bifurcations and instabilities in sliding Couette flow. JOURNAL OF FLUID MECHANICS 2011, 678: 156-178

ISSUE DATE:

2011-04-19

URL:

http://hdl.handle.net/2433/163439

RIGHT:

(C) Cambridge University Press 2011 


\section{Journal of Fluid Mechanics}

http://journals.cambridge.org/FLM

Additional services for Journal of Fluid Mechanics:

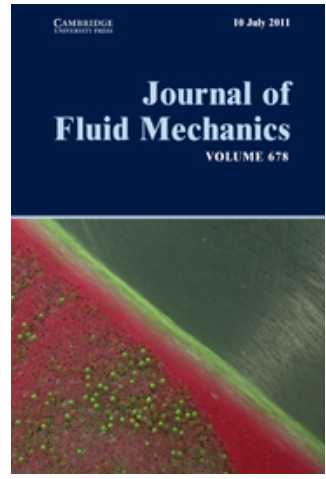

Email alerts: $\underline{\text { Click here }}$

Subscriptions: Click here

Commercial reprints: Click here

Terms of use : $\underline{\text { Click here }}$

\section{Bifurcations and instabilities in sliding Couette flow}

K. DEGUCHI and M. NAGATA

Journal of Fluid Mechanics / Volume 678 / July 2011, pp 156 - 178

DOI: 10.1017/jfm.2011.103, Published online: 19 April 2011

Link to this article: http://journals.cambridge.org/abstract_S0022112011001030

How to cite this article:

K. DEGUCHI and M. NAGATA (2011). Bifurcations and instabilities in sliding Couette flow. Journal of Fluid Mechanics,678, pp 156-178 doi:10.1017/jfm.2011.103

Request Permissions : $\underline{\text { Click here }}$ 


\title{
Bifurcations and instabilities in sliding Couette flow
}

\author{
K. DEGUCHI AND M. NAGATA $\dagger$ \\ Department of Aeronautics and Astronautics, Graduate School of Engineering, \\ Kyoto University, Kyoto 606-8501, Japan
}

(Received 10 March 2010; revised 4 February 2011; accepted 23 February 2011; first published online 19 April 2011)

We carry out linear and nonlinear analyses on a flow between two infinitely long concentric cylinders with the radii $a$ and $b$ subject to a sliding motion of the inner cylinder in the axial direction. We confirm the linear stability result of Gittler (Acta Mechanica, vol. 101, 1993, p. 1) for the axisymmetric case, namely the flow is linearly stable against axisymmetric perturbations when the radius ratio $\eta=a / b$ is greater than 0.1415 . We extend his analysis to the non-axisymmetric case and find that the stability of the flow is still determined by axisymmetric perturbations. Our nonlinear analysis exhibits that (i) finite-amplitude axisymmetric solutions exist far below the linear critical Reynolds number for $\eta<0.1415$ and (ii) non-axisymmetric travelling wave solutions appear abruptly at a finite Reynolds number even for $\eta>0.1415$ where the linear critical state is absent.

Key words: bifurcation, nonlinear instability, transition to turbulence

\section{Introduction}

Sliding Couette flow (SCF), classified by Joseph (1976), is a flow between two concentric cylinders where the cylinders mutually slide in the axial direction. SCF occurs in a wide range of situations in obvious engineering and medical contexts, such as a train moving through a tunnel and a catheter injected in a blood vessel. However, the basic academic research on SCF has not been intensively studied despite its simple geometry.

The linear stability of SCF was first studied by Preziosi \& Rosso (1990). They did not find any indication of instability within the parameter range they considered. However, Gittler (1993), restricting his analysis to axisymmetric perturbations, detected instability for $\eta \leqslant 0.1415$ at the Reynolds number which exceeded the range of Preziosi \& Rosso (1990) considerably.

$\mathrm{SCF}$, where the axial pressure gradient is absent, is a special case of annular Couette-Poiseuille flow. Annular Couette-Poiseuille flow has many industrial and medical applications: pipelinings (Arney et al. 1993), wire coating (Tadmor \& Bird 1974), production of optical fibres during the draw process (Panoliaskos, Hallett \& Garis 1985; Vaskopulos, Polymeropoulos \& Zebib 1993) and medical treatment by a thread injection (Frei, Lüscher \& Wintermantel 2000; Walton 2003, 2004, 2005). The effect of the sliding motion on the annular Poiseuille flow was examined by Sadeghi $\&$ Higgins (1991). They reported that for $\eta=0.5$ the neutral curves for the mode with the azimuthal wavenumbers $m_{0}=0,1$ and 2 receded to infinity when the sliding 


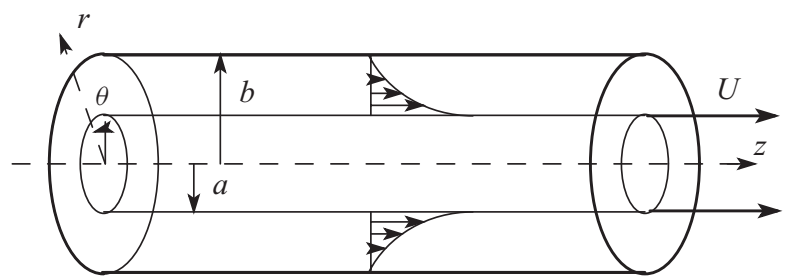

FIGURE 1. The configuration of sliding Couette flow.

velocity was approximately 35,65 and $25 \%$, respectively, of the maximum Poiseuille velocity. The asymmetric mode was generally the least stable as $\eta \rightarrow 0$.

If it is true that for the pure SCF the linear critical Reynolds number is infinite when $\eta>0.1415$, secondary flows, if they exist, must emerge abruptly, being disconnected from the laminar state. The absence of a linear critical state similarly occurs in other problems, such as plane Couette flow (PCF), pipe Poiseuille flow (PPF) and square duct flow (SDF). Finite-amplitude solutions for these flows have been extensively explored with success in recent years. (The discoveries for these solutions are by Nagata 1990 for PCF, Faisst \& Eckhardt 2003 and Wedin \& Kerswell 2004 for PPF and Wedin, Bottaro \& Nagata 2009; Okino et al. 2010 and Uhlmann, Kawahara \& Pinelli 2010 for SDF. Also, see Gibson, Halcrow \& Cvitanovic 2009 for PCF, Pringle, Duguet \& Kerswell 2009 for PPF and Okino \& Nagata 2011 for SDF, for a list of solutions yet found.) In contrast, SCF has attracted much less attention. No finite-amplitude solutions of SCF are known yet. Although a few experimental investigations were reported for annular Couette-Poiseuille flow (Shands, Alfredsson \& Lindgren 1980; Frei et al. 2000), they did not treat the case of the zero axial pressure gradient.

We formulate the problem in $\S 2$, followed by the linear stability analysis with respect to an arbitrary three-dimensional perturbation in $\S 3$. We confirm the result by Gittler (1993), i.e. SCF is linearly stable against axisymmetric perturbations for $\eta \leqslant 0.1415$. In addition, we find that the critical state is determined by axisymmetric perturbations. Axisymmetric finite-amplitude solutions that bifurcate from the linear critical state for $\eta \leqslant 0.1415$ are obtained in our nonlinear analysis in $\S 4$. These solutions exist at extremely high Reynolds numbers. In $\S 4$ we explore the possibility that other types of finite-amplitude solutions exist at lower Reynolds numbers. Using the fact that SCF approaches PCF in the narrow gap limit, we continue the finite-amplitude solutions of PCF (Nagata 1990) to wider gap cases. As a result, a family of finite-amplitude non-axisymmetric solutions is found at relatively low Reynolds numbers. These nonaxisymmetric solutions take the form of travelling waves propagating in the axial direction. In $\S 5$, the linear stability of the travelling waves obtained is examined. Finally a short summary is added in $\S 6$.

\section{Formulation of the problem}

\subsection{The governing equations and the basic state}

We consider an incompressible viscous fluid with the kinematic viscosity $v$ between two infinitely long concentric cylinders with radii $a$ and $b(b>a)$. The fluid experiences a shear motion when the inner cylinder is pulled with the axial speed $U$ while the outer cylinder is kept at rest. We take the cylindrical coordinates $(r, \theta, z)$ as shown in figure 1. To non-dimensionalize the problem, we use $(b-a) / 2,(b-a)^{2} / 4 v$ and $4 \rho v^{2} /(b-a)^{2}$ as the length, time and pressure scales, respectively. These length and time scales reduce the velocity scale to $2 v /(b-a)$, which is consistent with the 
one chosen by Nagata (1990) for PCF. In $\S 4.3$, the nonlinear solutions obtained by Nagata (1990) are continued to the SCF geometry. This choice of the velocity scale is also useful when one considers an extension to the Couette-Poiseuille flow problem, which is controlled by two independent velocities. The non-dimensional velocity $\boldsymbol{u}=u \boldsymbol{e}_{r}+v \boldsymbol{e}_{\theta}+w \boldsymbol{e}_{z}$, where $\boldsymbol{e}_{r}, \boldsymbol{e}_{\theta}$ and $\boldsymbol{e}_{z}$ are the unit vectors in the radial, azimuthal and axial directions, and the pressure $p$ are governed by

$$
\begin{aligned}
\nabla \cdot \boldsymbol{u} & =0, \\
\frac{\partial \boldsymbol{u}}{\partial t}+(\boldsymbol{u} \cdot \nabla) \boldsymbol{u} & =-\nabla p+\nabla^{2} \boldsymbol{u},
\end{aligned}
$$

subject to the no-slip boundary condition on the inner and outer cylinders at $r=r_{i}$ and $r=r_{o}$, respectively:

$$
\begin{array}{ll}
\boldsymbol{u}=R \boldsymbol{e}_{z} & \text { at } r=r_{i}=2 \eta /(1-\eta), \\
\boldsymbol{u}=\mathbf{0} & \text { at } \quad r=r_{o}=2 /(1-\eta),
\end{array}
$$

where $R=U(b-a) / 2 v$ is the Reynolds number and $\eta=a / b$. This system is equivalent under axial and azimuthal translations and azimuthal reflection.

The axial basic flow $\boldsymbol{U}_{B}$ depending only on $r$,

$$
\boldsymbol{U}_{B}(r)=U_{B}(r) \boldsymbol{e}_{z}=R \frac{\ln (r(1-\eta) / 2)}{\ln (\eta)} \boldsymbol{e}_{z},
$$

can be obtained as an exact solution of the problem.

\subsection{The disturbance equations}

We assume that the flow is periodic in the azimuthal and axial directions with the wavenumbers $m_{0}$ and $\alpha$, respectively. The flow is decomposed into a spatially varying fluctuation part $\tilde{\boldsymbol{u}}=\tilde{u} \boldsymbol{e}_{r}+\tilde{v} \boldsymbol{e}_{\theta}+\tilde{w} \boldsymbol{e}_{z}$ and a spatial mean part $\overline{\boldsymbol{u}}=\bar{u} \boldsymbol{e}_{r}+\overline{\boldsymbol{v}} \boldsymbol{e}_{\theta}+\bar{w} \boldsymbol{e}_{z}$. The latter is defined by the $(\theta, z)$-average:

$$
\overline{-}=\frac{m_{0} \alpha}{4 \pi^{2}} \int_{0}^{2 \pi / m_{0}} \int_{0}^{2 \pi / \alpha} \cdot \mathrm{d} \theta \mathrm{d} z .
$$

Notice that $\bar{u}$ vanishes by (2.1) with the boundary condition and that $\bar{w}$ includes $\boldsymbol{U}_{B}$. Following Marques (1990), the fluctuation part $\tilde{\boldsymbol{u}}$ is further decomposed into the poloidal part $\nabla \times \nabla \times\left(\phi \boldsymbol{e}_{r}\right)$ and the toroidal part $\nabla \times\left(\psi \boldsymbol{e}_{r}\right)$, so that the total velocity field $\boldsymbol{u}$ is expressed as

$$
\boldsymbol{u}=\bar{v} \boldsymbol{e}_{\theta}+\bar{w} \boldsymbol{e}_{z}+\nabla \times \nabla \times\left(\phi \boldsymbol{e}_{r}\right)+\nabla \times\left(\psi \boldsymbol{e}_{r}\right) .
$$

With this expression the equation of continuity (2.1) is satisfied. The equations for $\phi$ and $\psi$ are obtained by operating $\boldsymbol{e}_{r} \cdot \nabla \times$ and $\boldsymbol{e}_{r} \cdot \nabla \times \nabla \times$, respectively, on the momentum equation (2.2):

$$
\begin{aligned}
-r\left(\partial_{t}-\grave{\Delta}\right) \Delta_{2} & \frac{\psi}{r}+\frac{2}{r^{2}} \partial_{r} \frac{\psi_{\theta \theta}}{r}+\frac{2}{r}\left(\partial_{t}-2 \Delta\right) \frac{\phi_{\theta z}}{r}+\left((r \bar{v})^{\prime} \partial_{z}-\bar{w}^{\prime} \partial_{\theta}\right) \Delta_{2} \frac{\phi}{r} \\
& +\overline{\boldsymbol{u}} \cdot \nabla_{c}\left(\frac{2}{r^{2}} \phi_{\theta z}-\Delta_{2} \psi\right)+\boldsymbol{e}_{r} \cdot \nabla \times\left(\tilde{\boldsymbol{u}} \cdot \nabla_{c} \tilde{\boldsymbol{u}}+\frac{\tilde{v}}{r} \boldsymbol{e}_{z} \times \tilde{\boldsymbol{u}}\right)=0
\end{aligned}
$$

and

$$
\begin{aligned}
& r\left(\partial_{t}-\grave{\Delta}\right)\left(\grave{\Delta} \Delta_{2} \frac{\phi}{r}+\frac{2}{r^{3}} \partial_{r} \frac{\phi_{\theta \theta}}{r}\right)-\frac{2}{r^{2}}\left(\partial_{r} \Delta \frac{\phi_{\theta \theta}}{r}-\frac{2}{r^{2}} \phi_{\theta \theta z z}\right)+\frac{2}{r}\left(\partial_{t}-2 \Delta\right) \frac{\psi_{\theta z}}{r} \\
& +\overline{\boldsymbol{u}} \cdot \nabla_{c}\left(r \Delta_{2} \Delta \frac{\phi}{r}+2 \partial_{r} \frac{\phi_{z z}}{r}+\frac{2}{r^{2}} \psi_{\theta z}\right)+\overline{\boldsymbol{u}}^{\prime} \cdot \nabla_{c} \Delta_{2} \frac{\phi}{r}-r \overline{\boldsymbol{u}}^{\prime \prime} \cdot \nabla_{c} \Delta_{2} \frac{\phi}{r}
\end{aligned}
$$




$$
\begin{aligned}
& -\frac{2}{r^{2}} \bar{v}^{\prime} \Delta_{2} \phi_{\theta}-\frac{3}{r^{3}} \bar{v} \phi_{\theta z z}+\frac{1}{r^{5}} \bar{v} \phi_{\theta \theta \theta}+\frac{2}{r} \bar{v} \Delta_{2} \psi_{z} \\
& +\boldsymbol{e}_{r} \cdot \nabla \times \nabla \times\left(\tilde{\boldsymbol{u}} \cdot \nabla_{c} \tilde{\boldsymbol{u}}+\frac{\tilde{v}}{r} \boldsymbol{e}_{z} \times \tilde{\boldsymbol{u}}\right)=0,
\end{aligned}
$$

where the prime' denotes the differentiation with respect to $r$ and

$$
\left.\begin{array}{rl}
\Delta_{2} & =\frac{1}{r^{2}} \partial_{\theta \theta}^{2}+\partial_{z z}^{2}, \\
\Delta & =\partial_{r r}^{2}+\frac{1}{r} \partial_{r}+\frac{1}{r^{2}} \partial_{\theta \theta}^{2}+\partial_{z z}^{2}, \\
\grave{\Delta} & =\partial_{r r}^{2}+\frac{3}{r} \partial_{r}+\frac{1}{r^{2}} \partial_{\theta \theta}^{2}+\partial_{z z}^{2}, \\
\nabla_{c} & =\boldsymbol{e}_{r} \partial_{r}+\boldsymbol{e}_{\theta} \frac{1}{r} \partial_{\theta}+\boldsymbol{e}_{z} \partial_{z} .
\end{array}\right\}
$$

In order to obtain the equations for $\bar{v}$ and $\bar{w}$, we take the $(\theta, z)$-average of the azimuthal and axial components, respectively, of (2.2):

$$
\begin{gathered}
\partial_{t} \bar{v}=r^{-2} \partial_{r}\left\{r^{2} \overline{\left(\Delta_{2} \phi\right)\left(\partial_{\theta} \partial_{r} r^{-1} \phi+\partial_{z} \psi\right)}+\left(r^{2} \bar{v}^{\prime}-r \bar{v}\right)\right\}, \\
\partial_{t} \bar{w}=r^{-1} \partial_{r}\left\{r \overline{\left(\Delta_{2} \phi\right)\left(r^{-1} \partial_{r} r \partial_{z} \phi-r^{-1} \partial_{\theta} \psi\right)}+r \bar{w}^{\prime}\right\} .
\end{gathered}
$$

The no-slip boundary condition becomes

$$
\bar{v}=\bar{w}=\phi=\partial_{r} \phi=\psi=0 \quad \text { at } r=r_{i} \quad \text { and } \quad r_{o} .
$$

\section{The linear stability analysis of the basic state}

\subsection{Numerical method}

When the deviations from the basic state are infinitesimal, the Reynolds stresses in (2.11) and (2.12) can be omitted to result in $\bar{v}=0$ and $\bar{w}=U_{B}$, i.e. $\overline{\boldsymbol{u}}=\boldsymbol{U}_{\boldsymbol{B}}$. Neglecting those terms that are quadratic in $\tilde{\boldsymbol{u}}$ in (2.8) and (2.9), we obtain the linearized disturbance equations

$$
\begin{aligned}
-r\left(\partial_{t}-\grave{\Delta}\right) \Delta_{2} \frac{\check{\psi}}{r}+ & \frac{2}{r^{2}} \partial_{r} \frac{\check{\psi}_{\theta \theta}}{r}+\frac{2}{r}\left(\partial_{t}-2 \Delta\right) \frac{\check{\phi}_{\theta z}}{r} \\
& +\left((r \bar{v})^{\prime} \partial_{z}-\bar{w}^{\prime} \partial_{\theta}\right) \Delta_{2} \frac{\check{\phi}}{r}+\overline{\boldsymbol{u}} \cdot \nabla_{c}\left(\frac{2}{r^{2}} \check{\phi}_{\theta z}-\Delta_{2} \check{\psi}\right)=0
\end{aligned}
$$

and

$$
\begin{aligned}
& r\left(\partial_{t}-\grave{\Delta}\right)\left(\grave{\Delta} \Delta_{2} \frac{\check{\phi}}{r}+\frac{2}{r^{3}} \partial_{r} \frac{\check{\phi}_{\theta \theta}}{r}\right)-\frac{2}{r^{2}}\left(\partial_{r} \Delta \frac{\check{\phi}_{\theta \theta}}{r}-\frac{2}{r^{2}} \check{\phi}_{\theta \theta z z}\right)+\frac{2}{r}\left(\partial_{t}-2 \Delta\right) \frac{\check{\psi}_{\theta z}}{r} \\
& +\overline{\boldsymbol{u}} \cdot \nabla_{c}\left(r \Delta_{2} \Delta \frac{\check{\boldsymbol{\phi}}}{r}+2 \partial_{r} \frac{\check{\phi}_{z z}}{r}+\frac{2}{r^{2}} \check{\psi}_{\theta z}\right)+\overline{\boldsymbol{u}}^{\prime} \cdot \nabla_{c} \Delta_{2} \frac{\check{\phi}}{r}-r \overline{\boldsymbol{u}}^{\prime \prime} \cdot \nabla_{c} \Delta_{2} \frac{\check{\phi}}{r} \\
& \quad-\frac{2}{r^{2}} \bar{v}^{\prime} \Delta_{2} \check{\phi}_{\theta}-\frac{3}{r^{3}} \bar{v} \check{\phi}_{\theta z z}+\frac{1}{r^{5}} \bar{v} \check{\phi}_{\theta \theta \theta}+\frac{2}{r} \bar{v} \Delta_{2} \check{\psi}_{z}=0,
\end{aligned}
$$

where we write

$$
\tilde{\boldsymbol{u}}=\nabla \times \nabla \times\left(\check{\phi} \boldsymbol{e}_{r}\right)+\nabla \times\left(\check{\psi} \boldsymbol{e}_{r}\right) .
$$




$\left(\eta, m_{0}, \alpha, R\right)$
$\left(0.1,0,0.6546,3.6 \times 10^{6}\right)$
$\left(0.1,0,0.6546,3.6 \times 10^{6}\right)$
$\left(0.1,0,0.6546,3.6 \times 10^{6}\right)$
$\left(0.01,1,2.0,4.0 \times 10^{7}\right)$
$\left(0.01,1,2.0,4.0 \times 10^{7}\right)$
$\left(0.01,1,2.0,4.0 \times 10^{7}\right)$

$L$
120
130
140
120
550
600

$$
\begin{aligned}
\operatorname{Re}(s) & =\sigma \\
-7.25876 & \times 10^{0} \\
-7.28065 & \times 10^{0} \\
-7.28189 & \times 10^{0} \\
1.99913 & \times 10^{5} \\
-4.69859 & \times 10^{4} \\
-4.69859 & \times 10^{4}
\end{aligned}
$$$$
\operatorname{Im}(s)=\gamma
$$$$
-2.26731 \times 10^{6}
$$$$
-2.26731 \times 10^{6}
$$$$
-2.26731 \times 10^{6}
$$$$
-6.50952 \times 10^{7}
$$$$
-1.72860 \times 10^{5}
$$$$
-1.72860 \times 10^{5}
$$

TABLE 1 . The eigenvalue $s$ determined by the linear stability analysis.

We expand $\check{\phi}$ and $\check{\psi}$ as a sum of weighted normal modes as

$$
\begin{aligned}
& \check{\phi}(x, \theta, z, t)=\sum_{l=0}^{\infty} \check{a}_{l}\left(1-x^{2}\right)^{2} T_{l}(x) \exp \left(\mathrm{i} m_{0} \theta+\mathrm{i} \alpha z+s t\right), \\
& \check{\psi}(x, \theta, z, t)=\sum_{l=0}^{\infty} \check{b}_{l}\left(1-x^{2}\right) T_{l}(x) \exp \left(\mathrm{i}_{0} \theta+\mathrm{i} \alpha z+s t\right),
\end{aligned}
$$

where $s=\sigma+\mathrm{i} \gamma$ is the (complex-valued) growth rate and $T_{l}(x)$ are the Chebyshev polynomials. The radial interval $\left[r_{i}, r_{o}\right]$ is mapped to $[-1,1]$ in $x$ by $x=r-r_{m}$ where $r_{m}=\left(r_{i}+r_{o}\right) / 2=(1+\eta) /(1-\eta)$ is the mean radius. The factors $\left(1-x^{2}\right)$ and $\left(1-x^{2}\right)^{2}$ in (3.4) and (3.5) are introduced in order for $\check{\phi}$ and $\check{\psi}$ to satisfy the boundary condition (2.13). The ansatz used in (3.4) and (3.5) allows us to apply the separation of variables and reduce the resulting equations in $x$ only. We substitute (3.4) and (3.5), after truncating the infinite series at $l=L$ for numerical purposes, into the linearized disturbance equations (3.1) and (3.2), which are then evaluated at the collocation points,

$$
x_{i}=\cos \left(\frac{\mathrm{i} \pi}{L+2}\right), \quad i=1, \ldots, L+1 .
$$

The resulting linear algebraic eigenvalue problem with the growth rate $s$ as the eigenvalue,

$$
D_{i j} \check{X}_{j}=s B_{i j} \check{X}_{j}, \quad \check{X}_{j} \in\left(\check{a}_{l}, \check{b}_{l}\right),
$$

is solved numerically by using LAPACK package ZGGEVX. The matrices $D_{i j}$ and $B_{i j}$ depend on $\eta, R, m_{0}$ and $\alpha$, and so does $s$, i.e. $s=s\left(\eta, R, m_{0}, \alpha\right)$. The azimuthal reflection symmetry allows us to restrict the linear stability analysis to $m_{0} \in \mathbb{Z}_{\geqslant 0}$ and $\alpha \in[0, \infty)$.

\subsection{Results}

Table 1 shows typical eigenvalues calculated at different truncation levels $L$. From the table, we find that $L=120$ is sufficient when $R=3.6 \times 10^{6}$ and $m_{0}=0$ (it will be shown later that this $R$ is close to the critical value), whereas $L=600$ is needed if the Reynolds number is ten times higher than the critical value and $m_{0} \neq 0$. For a given $\eta$, the stability of the basic state at $R$ subject to a perturbation with $m_{0}$ is determined by the largest real part of $\sigma$ of the growth rate $s$ over all possible values of $\alpha$, which we denote by $\sigma_{\alpha}$. For $m_{0}=0$ and 1 , we plot $\sigma_{\alpha}$ on the $(\eta, R)$ plane in figure 2, where the truncation level $L=600$ is used. As shown in figure 2(a) for axisymmetric perturbations $\left(m_{0}=0\right)$, two eigenmodes are involved in the contour plot of $\sigma_{\alpha}$ in the $(\eta-R)$ plane: one has always negative $\sigma_{\alpha}$ and the other make the flow unstable above the locus of $\sigma_{\alpha}=0$ (thick solid curve). For the non-axisymmetric perturbation 

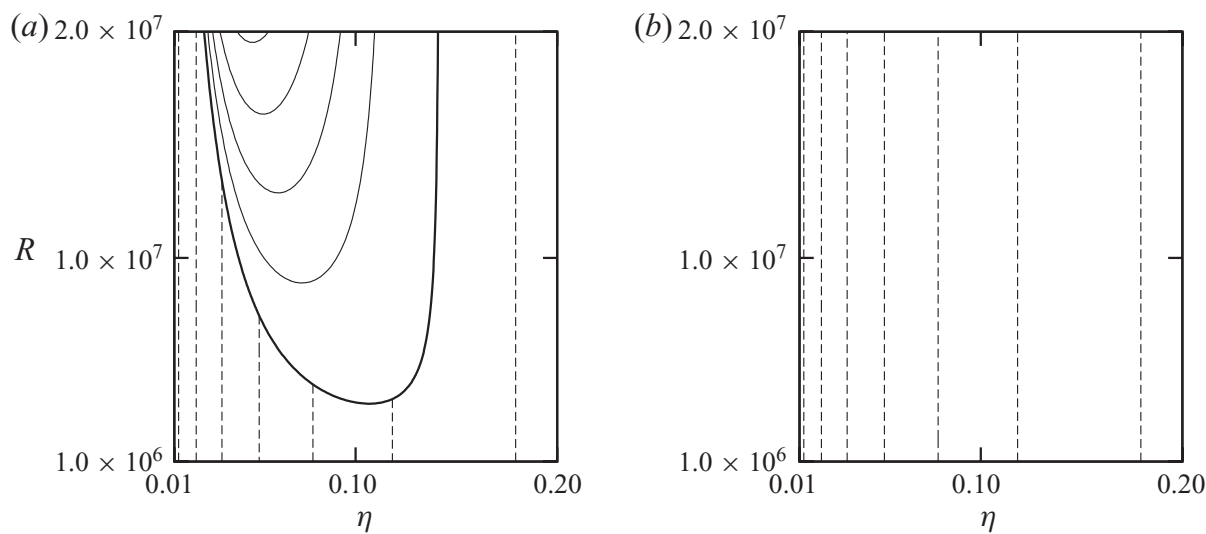

Figure 2. Contour plot of the largest real part $\sigma_{\alpha}$ of the eigenvalue $s$ as a function of the radius ratio $\eta=a / b$ and the Reynolds number $R$. (a) The dashed lines correspond to -8.2 , $-8.4,-8.5,-8.6,-8.8,-9.0$ and -9.2 (from left to right), the thick solid curve corresponds to 0 and the thin solid curves correspond to 4000, 8000, 12000 and 16000 (from bottom to top). ( $b$ ) The dashed lines correspond to $-8.2,-8.4,-8.5,-8.6,-8.8,-9.0$ and -9.2 (from left to right).
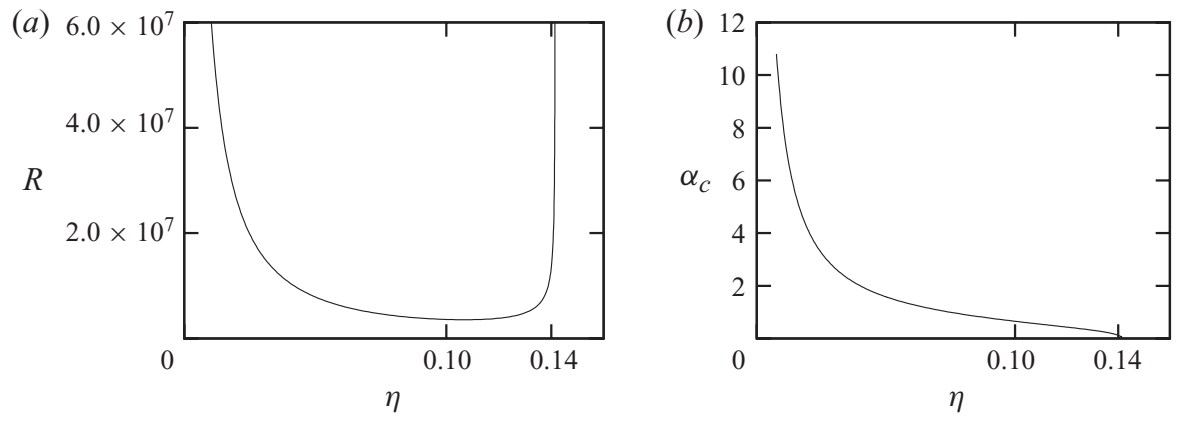

FIGURE 3. The neutral curve determined by axisymmetric perturbations $\left(m_{0}=0\right)$. $(a)$ The critical Reynolds number $R_{c}$. $(b)$ The critical axial wavenumber $\alpha_{c}$.

$\left(m_{0}=1\right), \sigma_{\alpha}$ is always negative as shown in figure $2(b)$. Other computations show that $\sigma_{\alpha}$ for $m_{0}=2,3,4$ also never reach zero. We, therefore, conclude that the flow is linearly stable to all non-axisymmetric perturbations. The locus of $\sigma_{\alpha}=0$ in figure 2(a) is reproduced in figure 3(a) as the neutral curve. We can see from figure $3(a)$ that as $\eta$ is decreased from the narrow gap limit $(\eta=1)$, the neutral curve emerges from infinity at $\eta \approx 0.14$ (more precisely, 0.1415 ), drops sharply reaching a minimum at $\eta \approx 0.1$ and ascends sharply towards infinity as $\eta$ approaches zero. The critical axial wavenumber $\alpha_{c}$ along the neutral curve varies as shown in figure $3(b)$. $\alpha_{c}$ increases monotonically from zero as $\eta$ decreases from 0.1415 . When $\eta=0.1, R_{c}=3.61 \times 10^{6}$ at $\alpha_{c}=0.6546$. This result is consistent with the findings of Gittler (1993), who restricted his linear stability analysis only to axisymmetric perturbations.

It can be proved that the inviscid SCF for any $\eta$ is stable for any axisymmetric perturbations when Rayleigh's inflection point theorem for the planar geometry is extended to the annular geometry (Problem 3.2 in Drazin \& Reid 1981; Walton 2004). Therefore, the fact that SCF for $\eta<0.1415$ becomes unstable implies that viscosity is necessary for linear instability. 
In the case of non-axisymmetric perturbations, $m_{0} \neq 0$, we find that $\sigma_{\alpha}$ is always determined by $\alpha=0$.

\section{The nonlinear analysis}

\subsection{Numerical method}

Assuming a travelling wave type of solution, $\phi, \psi, \bar{v}, \bar{w}$ are expanded as

$$
\begin{aligned}
\phi(x, \theta, z, t) & =\sum_{l=0}^{\infty} \sum_{\substack{m=-\infty \\
(m, n) \neq(0,0)}}^{\infty} \sum_{n=-\infty}^{\infty} a_{l m n} f_{l}(x) \exp \left(\mathrm{imm}_{0}\left(\theta-c_{\theta} t\right)+\mathrm{i} n \alpha\left(z-c_{z} t\right)\right), \\
\psi(x, \theta, z, t) & =\sum_{l=0}^{\infty} \sum_{\substack{m=-\infty \\
(m, n) \neq(0,0)}}^{\infty} \sum_{n=-\infty}^{\infty} b_{l m n} g_{l}(x) \exp \left(\mathrm{imm}_{0}\left(\theta-c_{\theta} t\right)+\mathrm{i} n \alpha\left(z-c_{z} t\right)\right), \\
\bar{v}(x) & =\sum_{l=0}^{\infty} d_{l} g_{l}(x=V(x), \\
\bar{w}(x) & =\sum_{l=0}^{\infty} e_{l} g_{l}(x)+U_{B}(x)=W(x)+U_{B}(x),
\end{aligned}
$$

where $c_{\theta}$ and $c_{z}$ are the phase velocities in the azimuthal and axial directions, respectively. The functions $f_{l}(x)$ and $g_{l}(x)$ in expansions (4.1)-(4.4) are $\left(1-x^{2}\right)^{2} T_{l}(x)$ and $\left(1-x^{2}\right) T_{l}(x)$, respectively. The amplitude coefficients, $a_{l m n}$ and $b_{l m n}$, are complex, whereas $d_{l}$ and $e_{l}$ are real. The reality condition for $\phi$ and $\psi$ implies

$$
a_{l m n}=a_{l-m-n}^{*}, \quad b_{l m n}=b_{l-m-n}^{*},
$$

where the asterisk indicates the complex conjugate.

We truncate the infinite series at $l=L,|m|=M,|n|=N$ and discretize (2.8)-(2.12) by the Galerkin method in the $\theta$ - and $z$-directions, whereas the collocation method with the collocation points defined by (3.6) is applied in the $x$-direction. We then obtain the nonlinear algebraic equation

$$
F_{i}=D_{i j} X_{j}+H_{i j k} X_{j} X_{k}+\mathrm{i}\left(m m_{0} c_{\theta}+n \alpha c_{z}\right) B_{i j} X_{j}=0,
$$

where $X_{j} \in\left(a_{l m n}, b_{l m n}, d_{l}, e_{l}\right)$. We include the phase velocities $c_{\theta}$ and $c_{z}$ as unknowns. The involvement of the additional variables, $c_{\theta}$ and $c_{z}$, for the nonlinear solution requires that the real or imaginary part of one- or two-amplitude coefficients, depending on the dimensionality of the solution, must be set to zero. This corresponds to 'freezing' the solution on the time coordinate. In our case, $\operatorname{Im}\left(a_{001}\right)=0$ is set for two-dimensional solutions and $\operatorname{Im}\left(a_{102}\right)=\operatorname{Im}\left(a_{120}\right)=0$ for three-dimensional ones.

We solve (4.6) by the Newton-Raphson iterative scheme with the tolerance

$$
\epsilon=\max _{j} \epsilon_{j}<10^{-5}
$$

where

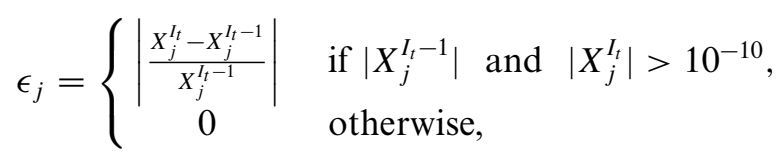

and $I_{t}$ is the iteration number. One might argue that the convergence criterion (4.6) is not very strong from the numerical analysis point of view. However, based on our 


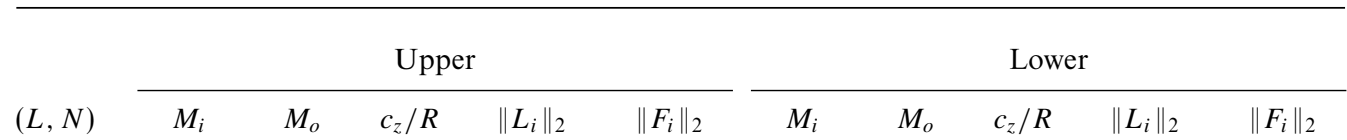

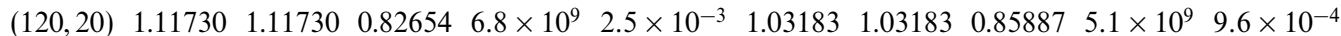
$\begin{array}{lllllllllll}(180,20) & 1.11730 & 1.11730 & 0.82654 & 8.6 \times 10^{9} & 7.1 \times 10^{-4} & 1.03183 & 1.03183 & 0.85890 & 5.5 \times 10^{9} & 5.3 \times 10^{-4}\end{array}$ $\begin{array}{lllllllllll}(120,30) & 1.11728 & 1.11727 & 0.82650 & 8.5 \times 10^{9} & 3.6 \times 10^{-4} & 1.03174 & 1.03174 & 0.85887 & 6.4 \times 10^{9} & 1.6 \times 10^{-3}\end{array}$

TABLE 2. The momentum transports $M_{i} / M_{o}$ on the inner/outer cylinders, the phase velocity $c_{z}$ and the $L_{2}$-norms $\left\|L_{i}\right\|_{2}$ and $\left\|F_{i}\right\|_{2}$ at different truncation levels $(L, N)$ for the axisymmetric solution at $(\eta, \alpha, R)=(0.1,0.6546,45000)$.

experiences in, for example, Nagata (1990) and Okino et al. (2010), we find that (4.6) is effective in facilitating the production of rather expensive calculations. The choice of $10^{-5}$ in (4.6) would be justified (see the Appendix).

The momentum transport on the inner cylinder at $r=r_{i}$, normalized by that of the base flow,

$$
M=M_{i}=\left.\frac{W^{\prime}+U_{B}^{\prime}}{U_{B}^{\prime}}\right|_{x=-1}
$$

is used as a measure of how much a nonlinear solution differs from the base flow. It can be verified by multiplying $r$ on (2.12) and integrating it in the $r$-direction so that the momentum transport on the outer cylinder at $r=r_{0}$, with the similar expression as above, namely $M_{o}=\left(W^{\prime}+U_{B}^{\prime}\right) /\left.U_{B}^{\prime}\right|_{x=1}$, is exactly equal to (4.9) although its numerical values differ slightly depending on the truncation level.

\subsection{Axisymmetric finite-amplitude solutions bifurcating from the linear critical point}

First, we explore axisymmetric finite-amplitude solutions which are expected to bifurcate at the linear critical point obtained in the previous section. To do this, we set $m_{0}=0$ and $\alpha=\alpha_{c}$ in (4.1) and (4.2). For $\eta=0.1$, the critical Reynolds number $R_{c}$ is $3.61 \times 10^{6}$ with $\alpha_{c}=0.6546$. It is found that for this $\eta$ the bifurcation is subcritical with a saddle-node at $R \approx 4.0 \times 10^{4}$ as shown in figure 4(a). Table 2 shows the variation of various measures $\left(M_{i}\right.$ and $M_{o}$ : the momentum transports on the inner and outer cylinders, respectively, $c_{z}$ : the axial phase speed and the $L_{2}$-norms of the linear part $L_{i}=D_{i j} X_{j}$ in (4.5), $\left\|L_{i}\right\|_{2}$, and the residual of (4.5), $\left\|F_{i}\right\|_{2}$ ) as a function of the truncation level $(L, N)$ for a typical axisymmetric solution. We see that the truncation level $(L, N)=(120,20)$ is sufficient for approximating the axisymmetric solutions. The momentum transport on the cylinders is smaller on the lower subcritical branch between the linear critical point and the saddle-node than that on the upper branch after the saddle-node. The phase speed $c_{z}$ decreases along the subcritical branch monotonically and continues to do so even after the saddle-node as shown in figure $4(b)$. Nonlinear calculations for other $\eta$ indicate that provided $\alpha$ is very small the subcritical bifurcation of the axisymmetric solutions extends even for $\eta>0.1415$, where the critical Reynolds number is infinite. The Reynolds number at the saddle-node points ranges roughly between $R=O\left(10^{3}\right)$ and $O\left(10^{4}\right)$. For example, if we fix $\alpha=0.03$, the saddle-node bifurcation occurs at $R=9.4 \times 10^{3}$ for $\eta=0.2$ and at $R=1.7 \times 10^{4}$ for $\eta=0.3$.

The fluctuation flow field of the subcritical axisymmetric solutions for $\eta=0.1$ at $R=45000$, near the saddle-node, has one localized flattened vortex ring attached to the inner cylinder as shown in figure $5(a)$. It is noticeable that the variations of the 

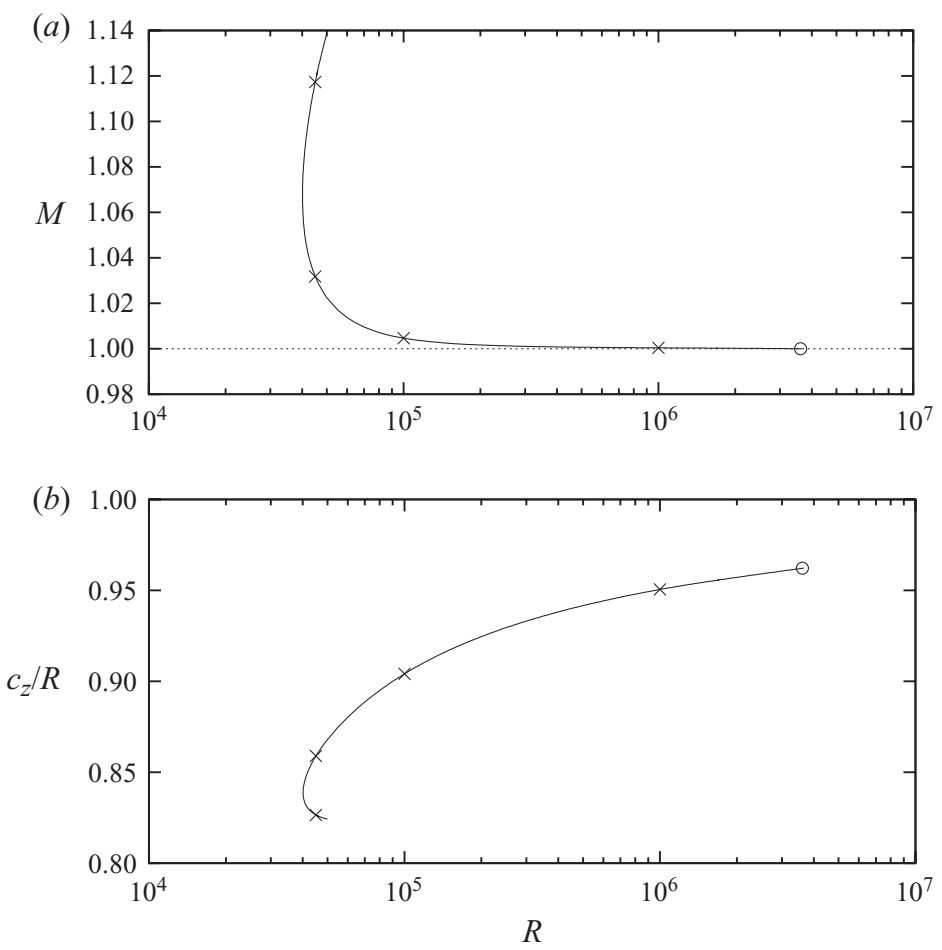

FIGURE 4. The subcritical bifurcation of the axisymmetric solution with $m_{0}=0$ and $\alpha=0.6546$ for $\eta=0.1 . R_{c}=3.61 \times 10^{6}$. The circles indicate the linear critical point. The measures of the nonlinearity are $(a)$ the momentum transport on the inner cylinder $M$ and $(b)$ the phase speed $c_{z}$. The truncation level $(L, N)=(120,20)$ is used to draw the curve, whereas crosses are plotted with $(L, N)=(180,30)$.

(a)
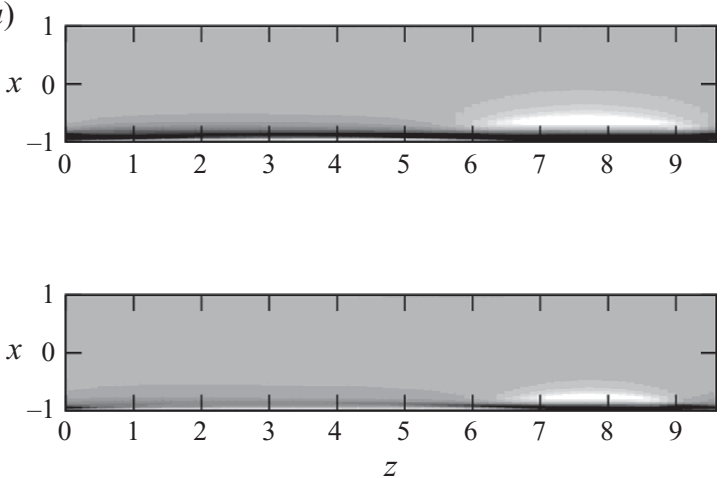

(b) $4.5 \times 10^{3}$

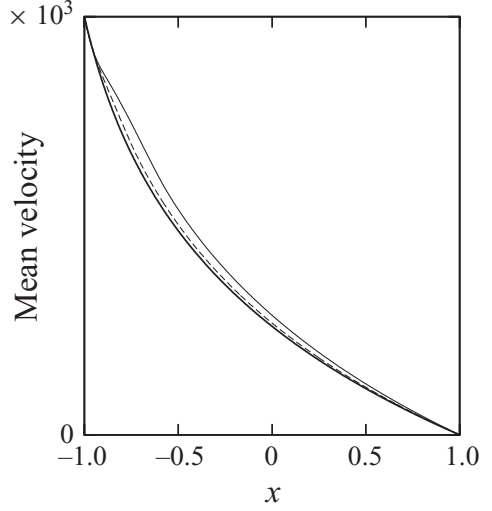

FIGURE 5. The flow field of the axisymmetric solution at $(R, \alpha)=(45000,0.6546)$. The truncation level $(L, N)=(120,20)$ is used. $(a)$ The azimuthal component of the vorticity relative to the laminar state $\omega_{\theta}-\omega_{\theta B}=(\partial u / \partial z)-\partial\left(w-U_{B}\right) / \partial r$ in grey scale (light: positive, dark: negative). The upper/lower figures correspond to the upper/lower branch solutions. (b) The mean flow profile. The solid and dashed curves represent the upper and lower branch solutions, respectively. The thick curve represents the base flow. 


$\begin{array}{lcc}(l, m, n) & a_{l m n}: \eta=0.9999 & a_{l m n}: \eta=1 \\ (0,1,1) & (-0.531545990,0.000342705) & (-0.531546101,0.000000000) \\ (0,1,2) & (0.015296839,-0.000020938) & (0.015296853,0.000000000) \\ (0,2,1) & (-0.000003429,-0.000523607) & (0.000000000,-0.000523609) \\ (0,2,2) & (0.000000662,0.000539484) & (0.000000000,0.000539484) \\ (1,1,1) & (0.000247443,0.381802584) & (0.000000000,0.381802665) \\ (1,1,2) & (0.000015775,0.013213093) & (0.000000000,0.013213102) \\ (1,2,1) & (-0.077862389,0.000048121) & (-0.077862404,0.000000000) \\ (1,2,2) & (0.004590099,-0.000005700) & (0.004590102,0.000000000) \\ (0,-1,1) & (0.531545990,-0.000342705) & (0.531546101,0.000000000) \\ (0,-1,2) & (0.015296839,-0.000020938) & (0.015296853,0.000000000) \\ (0,-2,1) & (0.000003429,0.000523607) & (0.000000000,0.000523609) \\ (0,-2,2) & (0.000000662,0.000539484) & (0.000000000,0.000539484) \\ (1,-1,1) & (-0.000247443,-0.381802584) & (0.000000000,-0.381802665) \\ (1,-1,2) & (0.000015775,0.013213093) & (0.000000000,0.013213102) \\ (1,-2,1) & (0.077862389,-0.000048121) & (0.077862404,0.000000000) \\ (1,-2,2) & (0.004590099,-0.000005700) & (0.004590102,0.000000000)\end{array}$

TABLE 3. The comparison of the leading-amplitude coefficients of the narrow gap SCF for $\eta=0.9999$ at $\left(\alpha, m_{0}\right)=(0.75,31168)$ and the corresponding coefficients of PCF $(\alpha, \beta)=(0.75,1.5585) . R=400$. The lower branch solution. $(L, M, N)=(20,6,4)$.

mean flows on both the lower and upper branches are slightly larger near the inner cylinder (see figure $5 b$ ).

\subsection{Non-axisymmetric travelling wave solutions continued from PCF limit}

In experiment, it is likely that the flow becomes turbulent at Reynolds numbers far below such very large values as we saw in the linear and nonlinear analyses with respect to axisymmetric disturbances. Therefore, we attempt to find other types of nonlinear solutions at relatively low Reynolds numbers. An obvious candidate for the new type would have some connection with the nonlinear solutions in PCF since the narrow gap limit between the cylinders corresponds to PCF. It is known that for PCF the critical Reynolds number is infinite. Yet, finite-amplitude steady three-dimensional solutions as a result of the saddle-node bifurcation at $R=O\left(10^{2}\right)$ exist (Nagata 1990). We anticipate that the three-dimensional solutions of PCF as our narrow gap limit $(\eta \rightarrow 1)$ still exist in wider gap cases $(\eta<1)$. We introduce the variable $y=r_{m} \theta$ and consider the limit as $\eta \rightarrow 1$. The base flow becomes

$$
U_{B}(x)=R(1-x) / 2 .
$$

In the expansions (4.1) and (4.2) the variable $\theta$ and the wavenumber $m_{0}$ are replaced by $y$ and $\beta=m_{0} / r_{m}$, respectively. First, we consider the case of $\eta=0.9999$ $\left(r_{m}=19999, m_{0}=31168\right.$ so that $\beta=1.5585$ for PCF) and use the steady threedimensional PCF solution with $(\alpha, \beta)=(0.75,1.5585)$ by Nagata $(1990)$ as the initial guess for the numerical iterations. The Newton-Raphson iteration successfully converges. The leading-amplitude coefficients for the converged solution at $\eta=0.9999$ are compared with the corresponding coefficients of the PCF solution at $R=400$ in table 3. The solution in the cylindrical coordinates inherits some features of the PCF solution: we can see from table 3 that

$$
\left.\begin{array}{ll}
a_{l m n}=-a_{l-m n} & \text { if } n \in \text { odd integers, } \\
a_{l m n}=a_{l-m n} & \text { if } n \in \text { even integers. }
\end{array}\right\}
$$



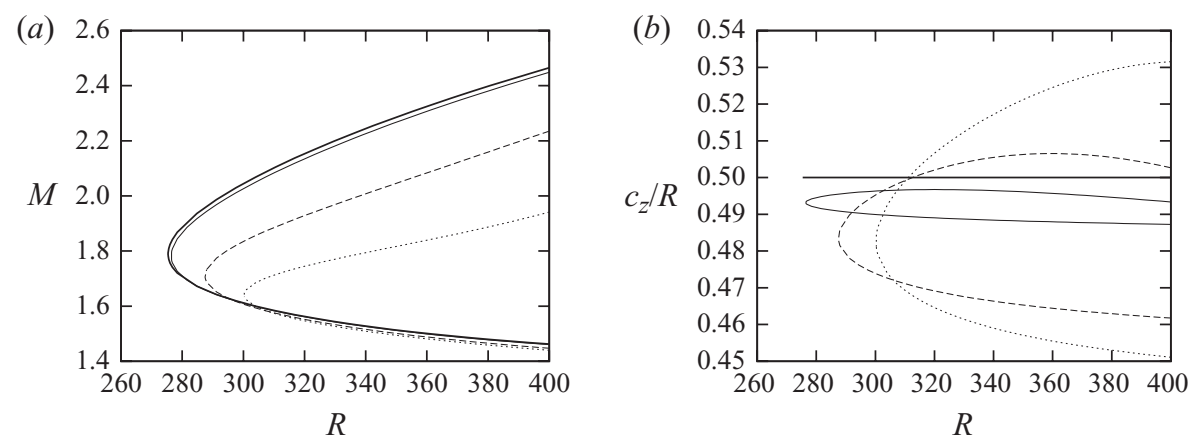

FIGURE 6. The saddle-node bifurcations of non-axisymmetric travelling wave solutions for $(\alpha, \beta)=(0.75,1.5585)$. The nonlinear measures are $(a)$ the momentum transport $M$ on the inner cylinder and $(b)$ the axial phase velocity $c_{z}$. The thick solid, thin solid, dashed and dotted curves indicate the cases for $\eta=1,0.8,0.5$ and 0.4 , respectively.

Not listed in table 3 we verify that

$$
\left.\begin{array}{ll}
b_{l m n}=b_{l-m n} & \text { if } n \in \text { odd integers, } \\
b_{l m n}=-b_{l-m n} & \text { if } n \in \text { even integers. }
\end{array}\right\}
$$

These relations imply the shift-reflection symmetry

$$
\text { (i) : }[u, v, w](x, y, z)=[u,-v, w](x,-y, z+\pi / \alpha) \text {, }
$$

which is possessed by PCF and SCF as well. It is known that the nonlinear PCF solution also possesses the shift-rotation symmetry

$$
\text { (ii) : }[u, v, w](x, y, z)=[-u, v,-w](-x, y+\pi / \beta,-z+\pi / \alpha) \text {. }
$$

Symmetry (ii) is obviously absent in SCF because the reflection about the mean radius $x=0$ corresponding to $r=r_{m}$ is broken due to the curvature in the cylindrical coordinates. Symmetry (ii) in PCF is represented by

$$
\left.\begin{array}{ll}
\operatorname{Im}\left(a_{l m n}\right)=\operatorname{Im}\left(b_{l m n}\right)=0 & \text { if } l+m \in \text { odd integers, } \\
\operatorname{Re}\left(a_{l m n}\right)=\operatorname{Re}\left(b_{l m n}\right)=0 & \text { if } l+m \in \text { even integers, }
\end{array}\right\}
$$

which is not satisfied in SCF as can be verified from table 3. So, for SCF with $\eta<1$ the nonlinear solution possesses only the symmetry (i): $[u, v, w](r, \theta, z)=$ $[u,-v, w](r,-\theta, z+\pi / \alpha)$.

Once the solution for $\eta=0.9999$ with $(\alpha, \beta)=(0.75,1.5585)$ is obtained, solutions for wider gap cases are sought by continuation. Changing $\eta$ implies that, according to $m_{0}=(1+\eta) \beta /(1-\eta), m_{0}$ does not necessarily take an integer value for fixed $\beta$. In order for the continuation to be smooth, we allow $m_{0}$ to take a real number at the first stage although physically $m_{0}$ must be an integer due to the $2 \pi$ azimuthal periodicity. This does not cause any computational difficulties. In the next stage, $2 \pi$ periodic solutions are sought for each $\eta$ by changing $\beta$ gradually so that $m_{0}$ becomes an integer.

Figure $6(a)$ shows the momentum transport $M$ of the solutions for $\eta=0.8,0.5$ and 0.4 with $(\alpha, \beta)=(0.75,1.5585)$. It is observed that the saddle-node points shift towards larger $R$ as $\eta$ is decreased. We see from figure $6(b)$ that the phase velocities $c_{z}$ in the axial direction for these cases are non-zero in general. The nonlinear solutions of PCF are steady when the two boundaries are moving in the opposite directions 


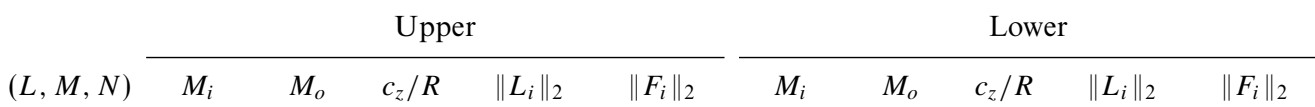

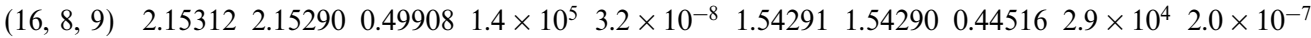

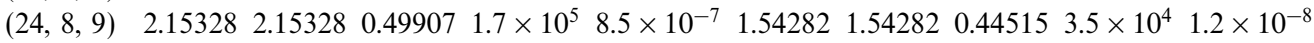
$\begin{array}{llllllllllll}(16,12,9) & 2.15067 & 2.15046 & 0.49976 & 1.4 \times 10^{5} & 2.7 \times 10^{-8} & 1.54277 & 1.54277 & 0.44514 & 2.9 \times 10^{4} & 1.4 \times 10^{-9}\end{array}$

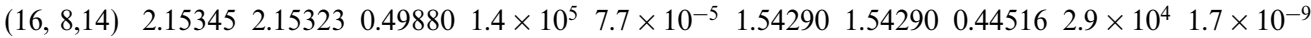

TABLE 4. The momentum transports $M_{i} / M_{o}$ on the inner/outer cylinders, the phase velocity $c_{z}$ and the $L_{2}$-norms $\left\|L_{i}\right\|_{2}$ and $\left\|F_{i}\right\|_{2}$ at different truncation levels $(L, M, N)$ for the non-axisymmetric solution at $\left(\eta, \alpha, m_{0}, R\right)=(0.5,0.51,3,350)$.

(a)

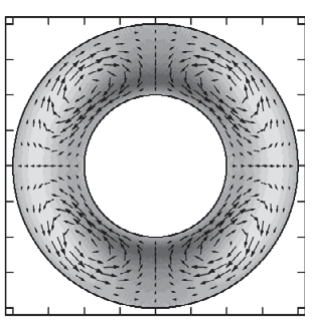

(b)

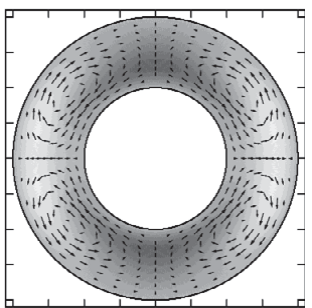

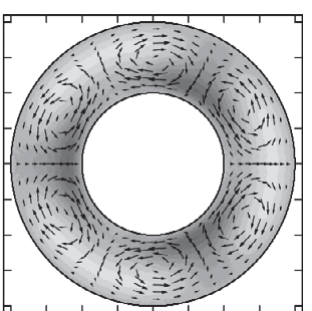

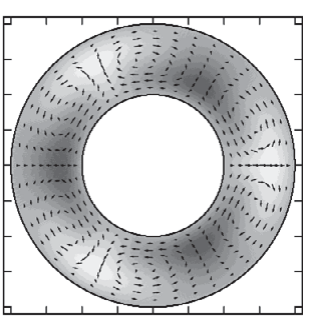

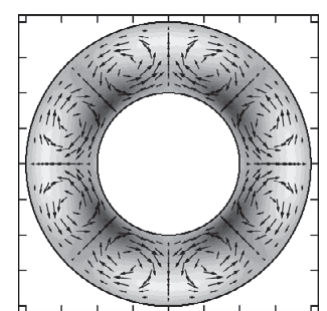

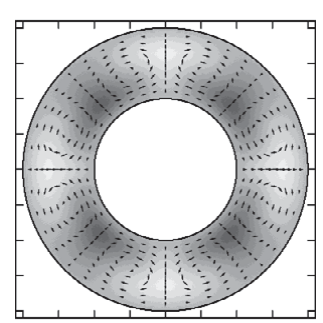

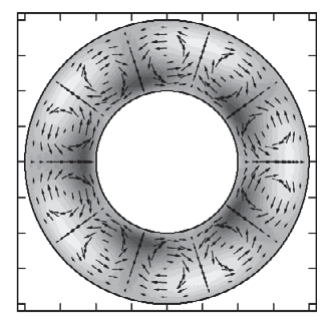

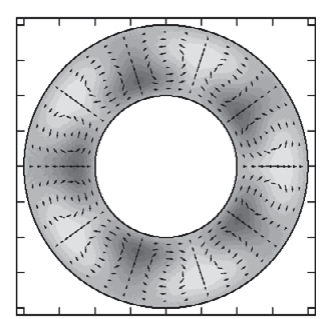

FIGURE 7. The axially averaged disturbance flow patterns for $\left(m_{0}, \alpha\right)=(2,0.33),(3,0.51)$, $(4,0.71),(5,0.90)$ (from left to right). $R=350$ and $\eta=0.5$. The four parts of $(a)$ and (b) correspond to the upper and lower branch solutions respectively. Grey scale represents the axial velocity component relative to the laminar state (light: fast, dark: slow).

with the same speed. Here, since the outer cylinder is stationary, the phase velocities $c_{z}$ of both the upper and lower branch solutions for $\eta=1$ are equal to half the speed of the inner cylinder. For wide gap cases, the phase velocity $c_{z}$ of the upper branch solution is faster than that of the lower branch solution in general. Consistent with the shift-reflection symmetry (i), our calculation showed that the phase velocity $c_{\theta}$ and the mean flow $V$ in the azimuthal direction are always zero.

\subsection{Results for $\eta=0.5$}

As described above, we change $\beta$ gradually to obtain non-axisymmetric travelling wave solutions of SCF with $m_{0}=2,3,4,5$ for $\eta=0.5$. Table 4 shows the accuracy of a typical solution at various truncation levels. We see $(L, M, N)=(16,8,9)$ giving an accuracy satisfactorily enough and adopt this truncation level for the calculations in this section unless stated otherwise. Figure 7 shows the axially averaged disturbance flow patterns for these solutions. Note that the patterns are still dependent on $\theta$. It can be seen that for all the cases the region where the axial velocity is faster than the basic flow is located near the outer cylinder, whereas the region with the axial velocity slower than the base flow is located near the inner cylinder. 


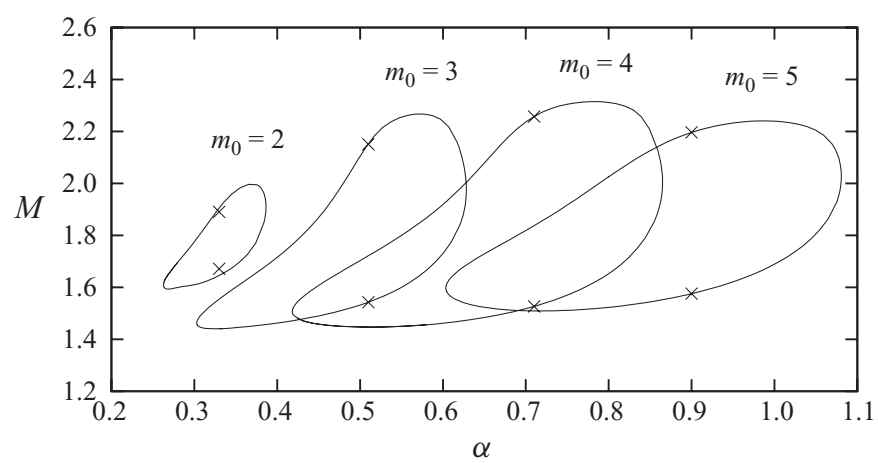

FIGURE 8 . The existence region of the travelling wave solutions for $m_{0}=2,3,4,5$ at $R=350$ for $\eta=0.5$. The truncation level $(L, M, N)=(16,8,9)$ is used to draw the curve. The crosses indicate the result with higher truncation level $(L, M, N)=(20,12,12)$.

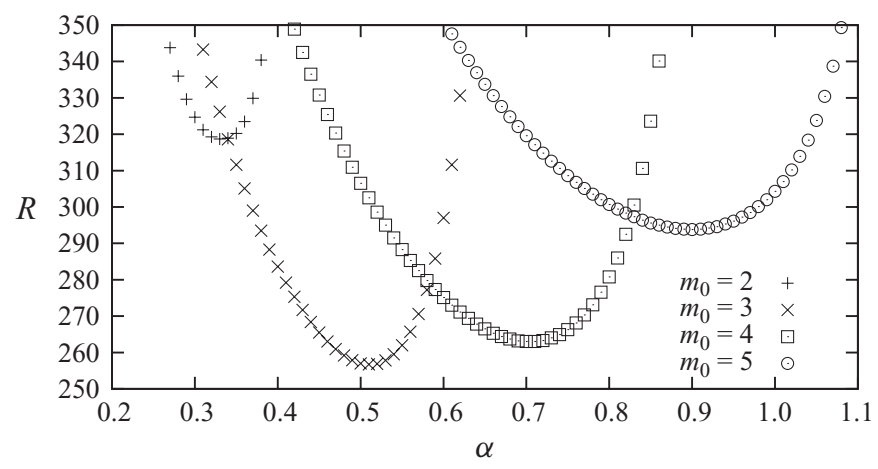

FiguRE 9. The Reynolds number near the saddle-node bifurcation point for the travelling wave solutions with the wavenumber pairs $\left(m_{0}, \alpha\right)$ for $\eta=0.5$.

Figure 8 shows the existence region of the travelling wave solutions with $m_{0}=2,3,4,5$ with respect to the axial wavenumber $\alpha$ at $R=350$. For other azimuthal wavenumbers, $m_{0}=1$ and $m_{0}=6, \ldots$, non-axisymmetric travelling wave solutions appear at higher Reynolds numbers, and so their existence regions are not captured in figure 8 . The solution family is created by the saddle-node bifurcation for each wavenumber pair $\left(m_{0}, \alpha\right)$ as shown in figure 9 . From the figure we see that the lowest Reynolds number at the saddle-node bifurcations takes place at 256.6 with $\left(m_{0}, \alpha\right)=(3,0.51)$. Recall that the linear critical state is absent for $\eta=0.5$. It is very likely that the lower branch solutions originate from the finite-amplitude streaky field at asymptotically large $R$ as described by Wang, Gibson \& Waleffe (2007) in the case of PCF. The axial mean flow profiles $\bar{w}=W+U_{B}$ are given in figure 10 . It is seen that as the Reynolds number increases the profile becomes sharper near the cylinders with larger momentum transport than in the central region. The flat central region becomes wider, especially for the upper branch.

The disturbance flow field $\boldsymbol{u}-\boldsymbol{U}_{B}$ of the travelling wave solution with $\left(m_{0}, \alpha\right)=$ $(3,0.51)$ for $\eta=0.5$ along the axial direction is shown in figure 11. It can be observed that slow speed streaks occupy the region near the inner cylinder, whereas fast speed streaks are located near the outer cylinder. The structure of the streaks along the axial direction is also depicted in figure 12. Sinusoidal variation of the fast and slow speed streaks can be seen. At $r=r_{m}$, the fast speed streaks are accompanied by the radially outward velocities, whereas the slow speed streaks are accompanied by the radially 

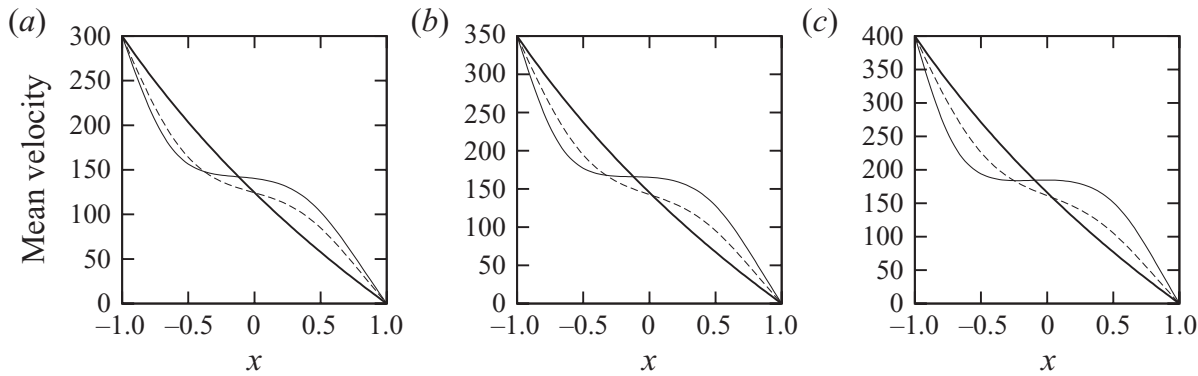

FiguRE 10. The axial mean flow profile $\bar{w}$ for $\left(\eta, m_{0}, \alpha\right)=(0.5,3,0.51) .(a) R=300,(b) R=350$ and (c) $R=400$. The thin solid and dashed curves indicate the mean flows for the upper and lower branch solutions, respectively. The thick curves correspond to the base flow.

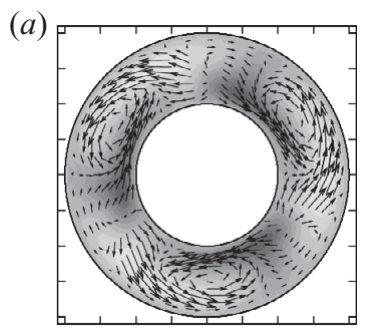

(b)

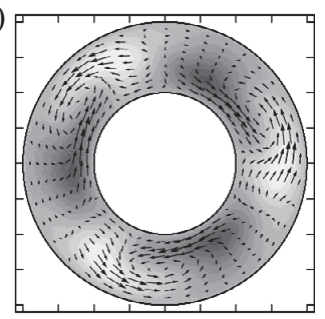

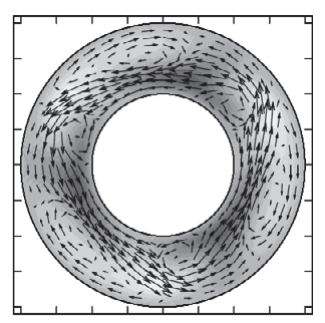

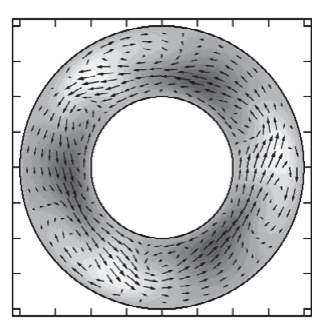

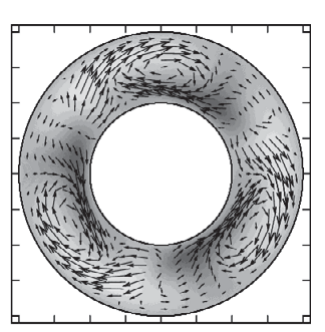

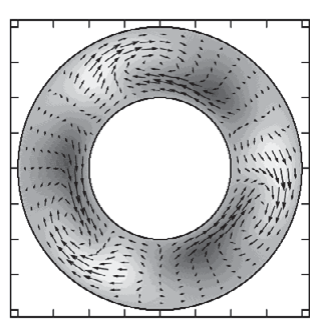

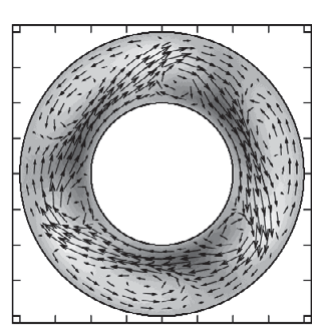

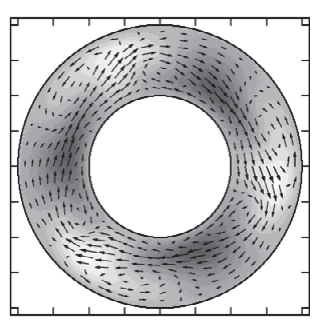

Figure 11. The disturbance flow field $\boldsymbol{u}-\boldsymbol{U}_{\boldsymbol{B}}$ for $\left(\eta, m_{0}, \alpha, R\right)=(0.5,3,0.51,350)$. The four parts of $(a)$ and $(b)$ correspond to the upper and lower branch solutions, respectively. The arrows indicate the projection of the velocity on the cross-section, $z=(k / 4)(2 \pi / \alpha), k=0,1,2,3$ (from left to right). Grey scale represents the axial velocity component (light: fast, dark: slow).

inward velocities. Figures $13(a)$ and $13(b)$ show the isosurfaces of the axial velocity component, $w-U_{B}$, and the axial vorticity component, $\omega_{z}=r^{-1} \partial_{r}(r v)-r^{-1} \partial_{\theta} u$, of the upper solution branch. Those corresponding to the lower solution branch are given in figures $13(c)$ and $13(d)$. By superposing figure $13(a)$ on figure $13(b)$ or figure $13(c)$ on figure $13(d)$, it can be seen that the streaks are flanked by stronger vorticities.

\subsection{Results for $\eta=0.1$}

The non-axisymmetric travelling wave solutions are continued to $\left(\eta, m_{0}\right)=(0.1,1)$. We find that the truncation level $(L, M, N)=(19,10,10)$, higher than in the case of $\eta=0.5$, is needed for $\eta=0.1$ (see table 5). The momentum transport $M$ of the continued solutions changes with $\alpha$ as indicated by the solid curves in figure 14. Some of the amplitude coefficients of the solution vanish at the points (circle), creating a new highly symmetric solution branch (dashed curve) as shown in figures 14(a) and $14(b)$. This new type of solution satisfies the symmetry

$$
\text { (iii) : }[u, v, w](x, \theta, z)=[u, v, w]\left(x, \theta+\pi / m_{0}, z+\pi / \alpha\right),
$$



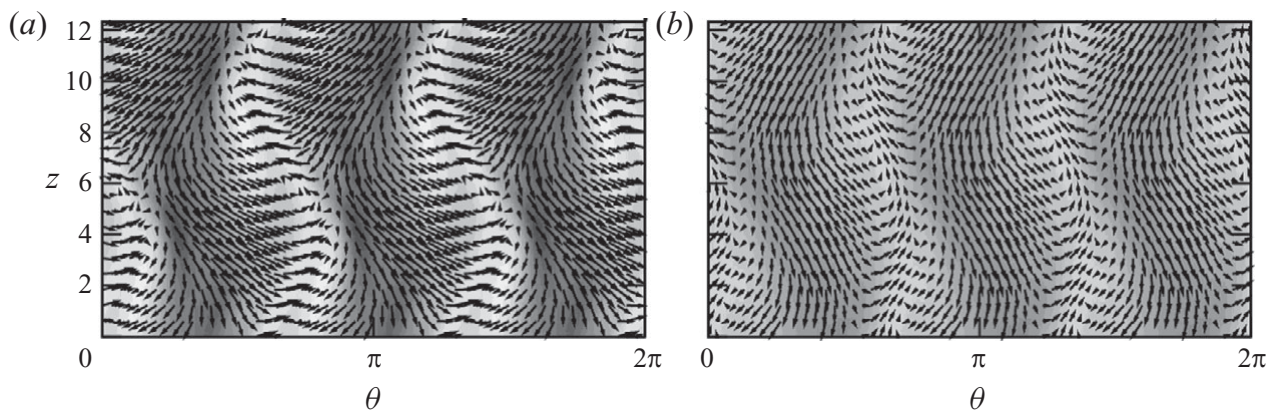

FIGURE 12. The disturbance flow field $\boldsymbol{u}-\boldsymbol{U}_{\boldsymbol{B}}$ for $\left(\eta, m_{0}, \alpha, R\right)=(0.5,3,0.51,350)$ on the mean radius $r=r_{m}$. Grey scale represents the radial velocity component. $(a)$ and $(b)$ correspond to the upper and lower branch solutions, respectively.
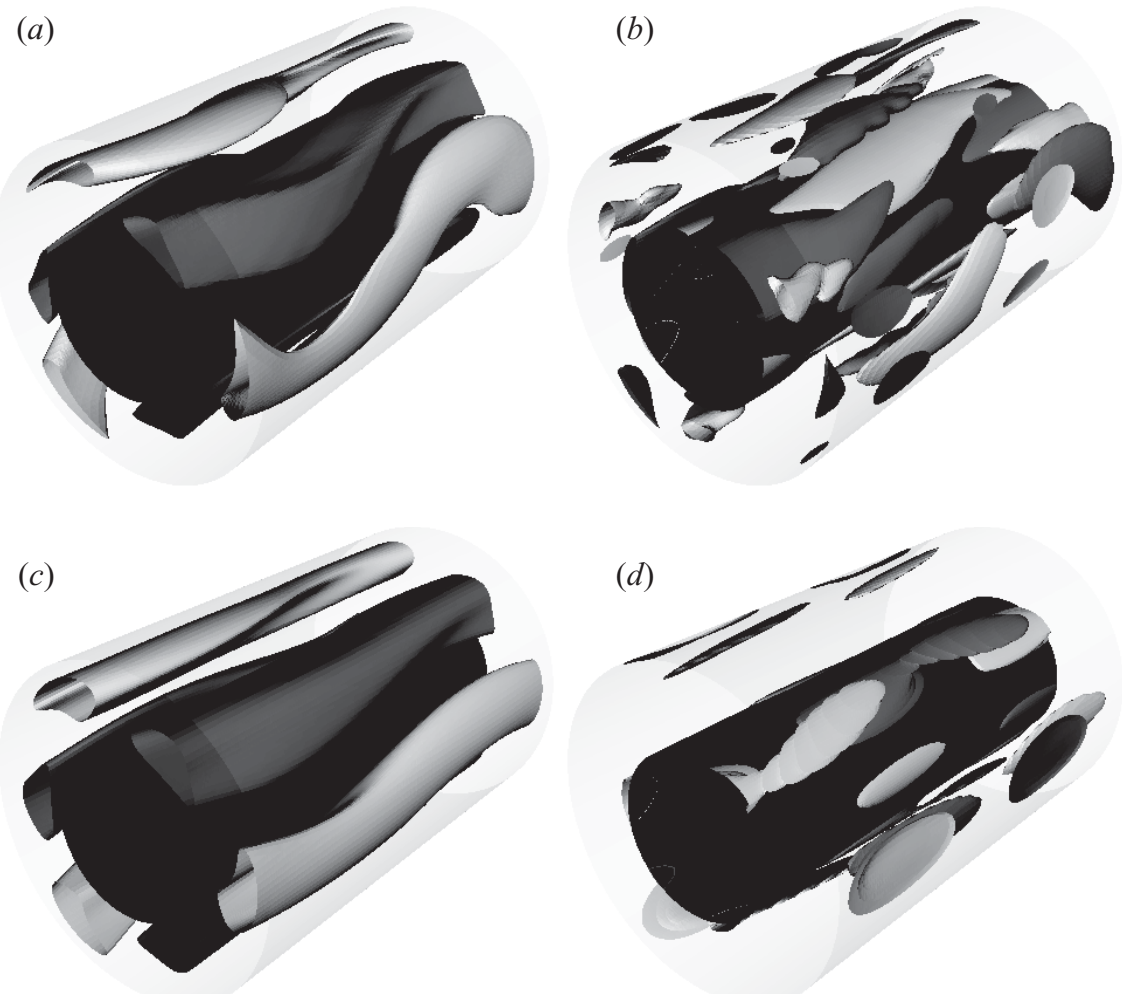

FIgURE 13. The axial variation of the streaks for the travelling wave for $\left(\eta, m_{0}, \alpha, R\right)=$ $(0.5,3,0.51,350) .(a),(c)$ The isosurface of the axial velocity at $w-U_{B}= \pm 70$. The light $/$ dark grey surfaces correspond to the fast/slow streaks. $(b),(d)$ The isosurface of the axial vorticity at $\omega_{z}= \pm 90$. The light/dark grey surfaces correspond to positive/negative vorticities. $(a)$ and $(b)$ correspond to the upper branch solution whereas $(c)$ and $(d)$ to the lower branch solution.

together with symmetry (i). The combined symmetry, (i) and (iii), represents the mirror symmetry

$$
\text { (i) }+ \text { (iii) : }[u, v, w](x, \theta, z)=[u,-v, w]\left(x,-\theta+\pi / m_{0}, z\right) \text {. }
$$

We call this type of solution the class $\mathscr{M}$ and the solution which is continued previously from PCF the class $\mathscr{P}$. The branches of the classes $\mathscr{P}$ and $\mathscr{M}$ solutions 


\begin{tabular}{|c|c|c|c|c|c|c|c|c|c|c|}
\hline \multirow[b]{2}{*}{$(L, M, N)$} & \multicolumn{5}{|c|}{ Upper } & \multicolumn{5}{|c|}{ Lower } \\
\hline & $M_{i}$ & $M_{o}$ & $c_{z} / R$ & $\left\|L_{i}\right\|_{2}$ & $\left\|F_{i}\right\|_{2}$ & $M_{i}$ & $M_{o}$ & $c_{z} / R$ & $\left\|L_{i}\right\|_{2}$ & $\left\|F_{i}\right\|_{2}$ \\
\hline & .56900 & 1.5690 & 0.41746 & $3.3 \times 10^{4}$ & $4.9 \times 10^{-8}$ & 1.41511 & 1.41512 & 0.35393 & $1.4 \times 10^{4}$ & $2.0 \times 10$ \\
\hline & 56901 & 1.569 & 0.41746 & $4.1 \times 10^{4}$ & $8.2 \times 10$ & 1.41511 & 1.41511 & 0.35393 & $1.7 \times 10^{4}$ & $6.9 \times 10^{-9}$ \\
\hline & 1.57075 & 1.57075 & 0.41717 & $3.4 \times 10^{4}$ & $5.3 \times 10^{-8}$ & 1.41715 & 1.41716 & 0.35426 & $1.4 \times 10^{4}$ & $2.4 \times 10^{-9}$ \\
\hline$(19,10,15)$ & 1.56880 & 1.56880 & 0.41751 & $3.4 \times 10^{4}$ & $4.6 \times 10^{-8}$ & 1.41511 & 1.41512 & 0.35393 & $1.4 \times 10^{4}$ & $3.4 \times 10^{-9}$ \\
\hline
\end{tabular}

TABLE 5. The momentum transports $M_{i} / M_{o}$ on the inner/outer cylinders, the phase velocity $c_{z}$ and the $L_{2}$-norms $\left\|L_{i}\right\|_{2}$ and $\left\|F_{i}\right\|_{2}$ at different truncation levels $(L, M, N)$ for the non-axisymmetric solution at $\left(\eta, \alpha, m_{0}, R\right)=(0.1,0.59,1,300)$.

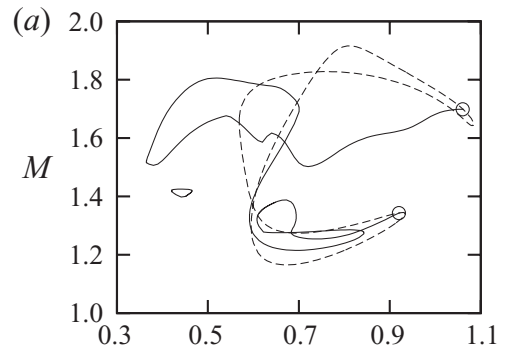

(b)
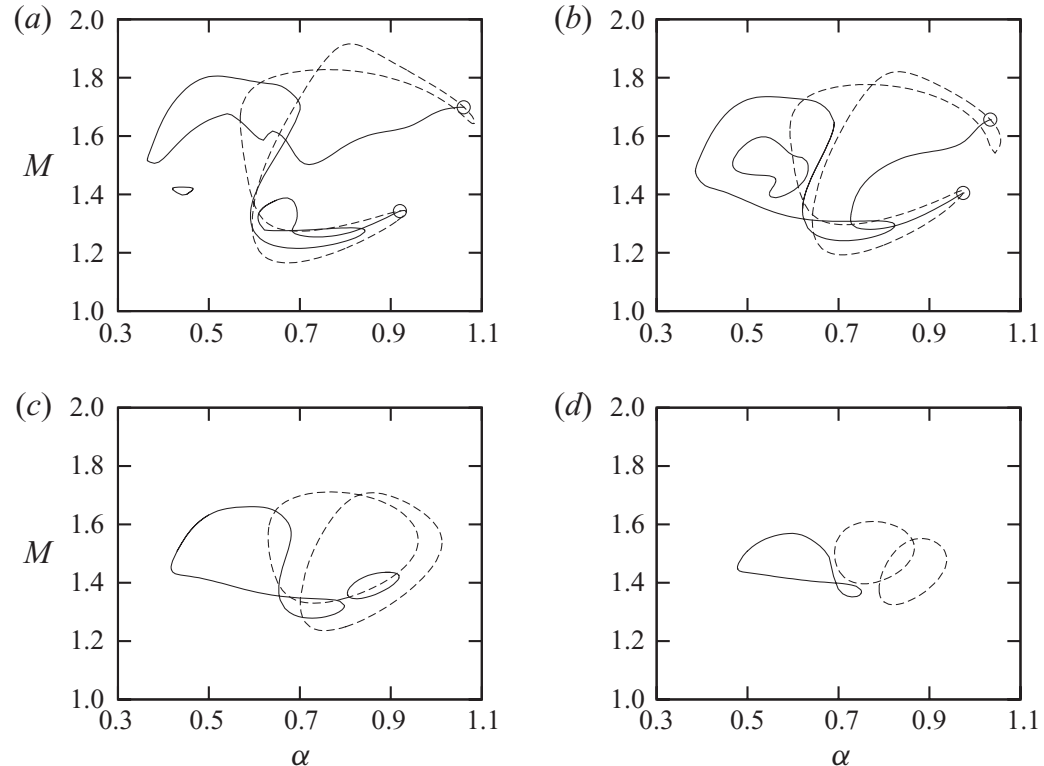

FIGURE 14. The momentum transport of the solutions for $\eta=0.1$. The solid/dashed curves represent the class $\mathscr{P} / \mathscr{M}$ solutions with $m_{0}=1$. (a) $R=360$, (b) $R=340$, (c) $R=320$, (d) $R=300$. The circles in $(a)$ and $(b)$ are the bifurcation points. The truncation level $(L, M, N)=(19,10,10)$ is used to draw the curve.

change their shapes in a very complex manner as $R$ is varied from 360 to 320 (see figures $14 a, 14 b$ and $14 c$ ). One closed curve of the class $\mathscr{P}$ and two closed curves of the class $\mathscr{M}$ are formed at $R=300$. Each of these closed curves in the $(\alpha, M)$ plane corresponds to the cross-sections of the 'nose' in the $(\alpha, R, M)$ space. The tip of the nose for the class $\mathscr{P}$ is located at $R=288.6$ with $\alpha=0.59$, and the tips of the two noses for the class $\mathscr{M}$ are at $R=291.8$ and 294.7 with $\alpha=0.77$ and 0.86 , respectively, as shown in figure 15. Thus, the first emerging state is the class $\mathscr{P}$ solution when $\eta=0.1$. The flow fields of the class $\mathscr{P}$ solution and the two class $\mathscr{M}$ solutions along the axial direction at $R=300$ are given in figure 16. For all the three solutions, low speed streaks stay near the inner cylinder almost without changing their positions, whereas fast speed streaks oscillate in the azimuthal direction. The regions of the most violent cross-sectional motion coincide with the regions of the fast speed streaks. The streaks 


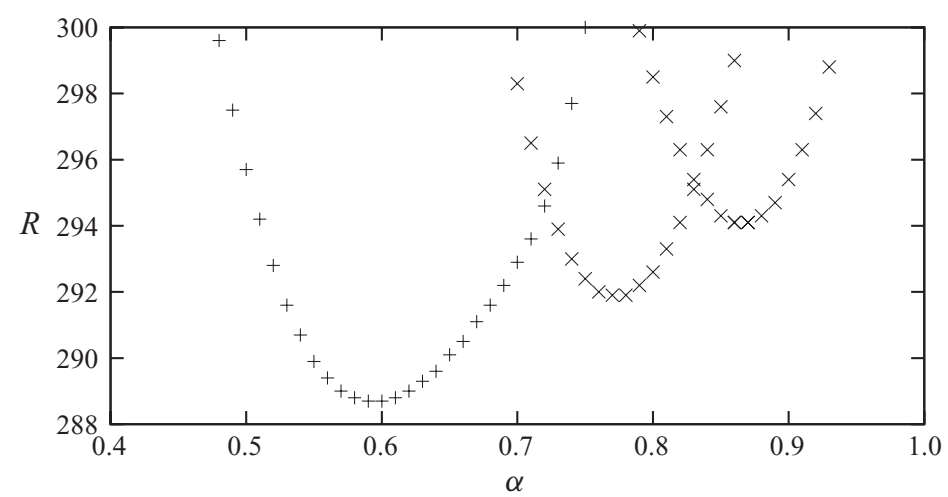

FIGURE 15. The saddle-node bifurcations for the non-axisymmetric solutions for $\eta=0.1 .+/ \times$ represents the class $\mathscr{P} / \mathscr{M}$ solutions with $m_{0}=1$.

and the axial vorticity for these solutions are given in figure 17, where the streaks are flanked by strong axial vortices.

For $m_{0}=2$, solutions of the class $\mathscr{M}$ and the class $\mathscr{P}$ begin to exist at Reynolds numbers about two times higher than those for $m_{0}=1$. Bifurcation points that connect the classes $\mathscr{P}$ and $\mathscr{M}$ have not been detected.

We note in passing that similar mirror-symmetric solutions are found in PPF (Pringle \& Kerswell 2007) and SDF (Okino et al. 2010) at low Reynolds numbers. These solutions have a large pair of vortices in the cross-section and asymmetric solutions bifurcate from them as in our case.

\section{The linear stability of the travelling waves}

In order to examine the stability of the travelling wave solutions described in the previous section, we superimpose an infinitesimal perturbation on the flow field. Here we restrict our attention to disturbances with the fundamental mode, namely the disturbance has the same wavenumbers and the phase velocities as the travelling wave solution. The total velocity field is now given by

$$
\begin{aligned}
\boldsymbol{u}=\boldsymbol{u}_{T W}+\check{\boldsymbol{u}}=\left(\bar{v}_{T W}+\overline{\check{v}}\right) \boldsymbol{e}_{\theta}+\left(\bar{w}_{T W}+\bar{w}\right) \boldsymbol{e}_{z}+\nabla \times \nabla & \times\left(\left(\phi_{T W}+\check{\phi}\right) \boldsymbol{e}_{r}\right) \\
& +\nabla \times\left(\left(\psi_{T W}+\check{\psi}\right) \boldsymbol{e}_{r}\right),
\end{aligned}
$$

where the subscript $T W$ denotes the travelling wave solution and the check denotes the superimposed infinitesimal perturbations. The perturbations $\check{\phi}, \check{\psi}, \check{v}, \check{w}$ are expanded as

$$
\begin{aligned}
\check{\phi}(x, \theta, z, t) & =\sum_{l=0}^{\infty} \sum_{\substack{m=-\infty \\
(m, n) \neq(0,0)}}^{\infty} \sum_{n=-\infty}^{\infty} \check{a}_{l m n} f_{l}(x) \exp \left(\mathrm{imm} m_{0}\left(\theta-c_{\theta} t\right)+\mathrm{i} n \alpha\left(z-c_{z} t\right)+s t\right), \\
\check{\psi}(x, \theta, z, t) & =\sum_{l=0}^{\infty} \sum_{\substack{m=-\infty \\
(m, n) \neq(0,0)}}^{\infty} \sum_{n=-\infty}^{\infty} \check{b}_{l m n} g_{l}(x) \exp \left(\mathrm{i}^{2} m_{0}\left(\theta-c_{\theta} t\right)+\mathrm{i} n \alpha\left(z-c_{z} t\right)+s t\right), \\
\bar{v}(x, t) & =\sum_{l=0}^{\infty} \check{d}_{l} g_{l}(x) \exp (s t),
\end{aligned}
$$


(a)
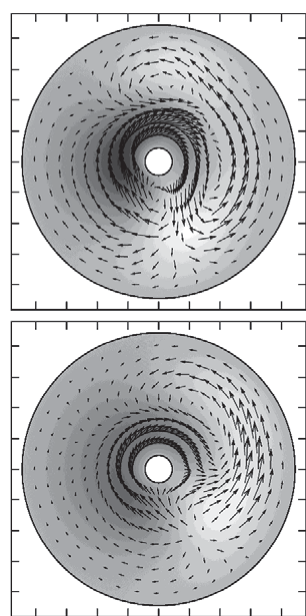

(b)
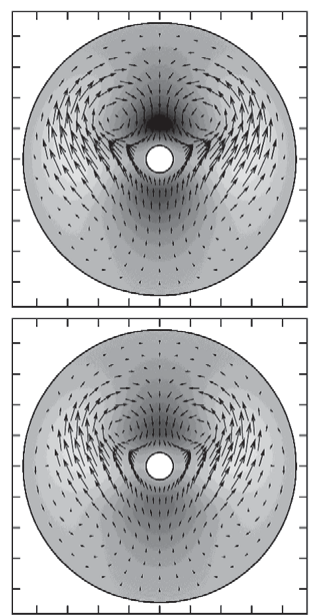

(c)
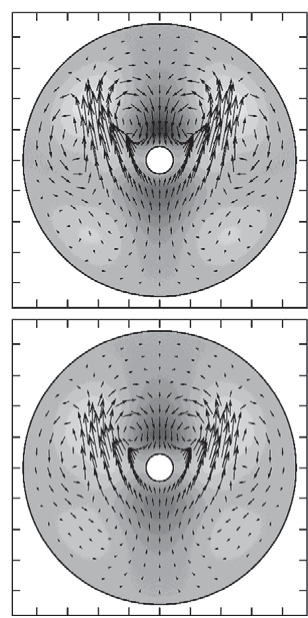
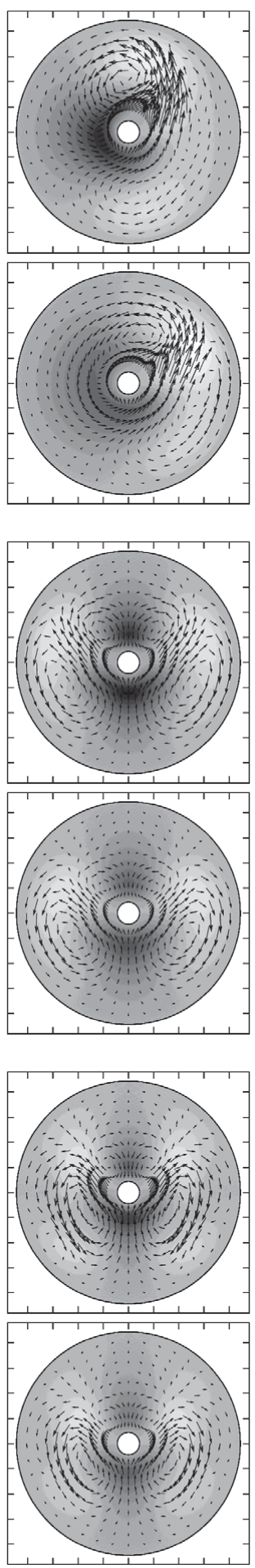
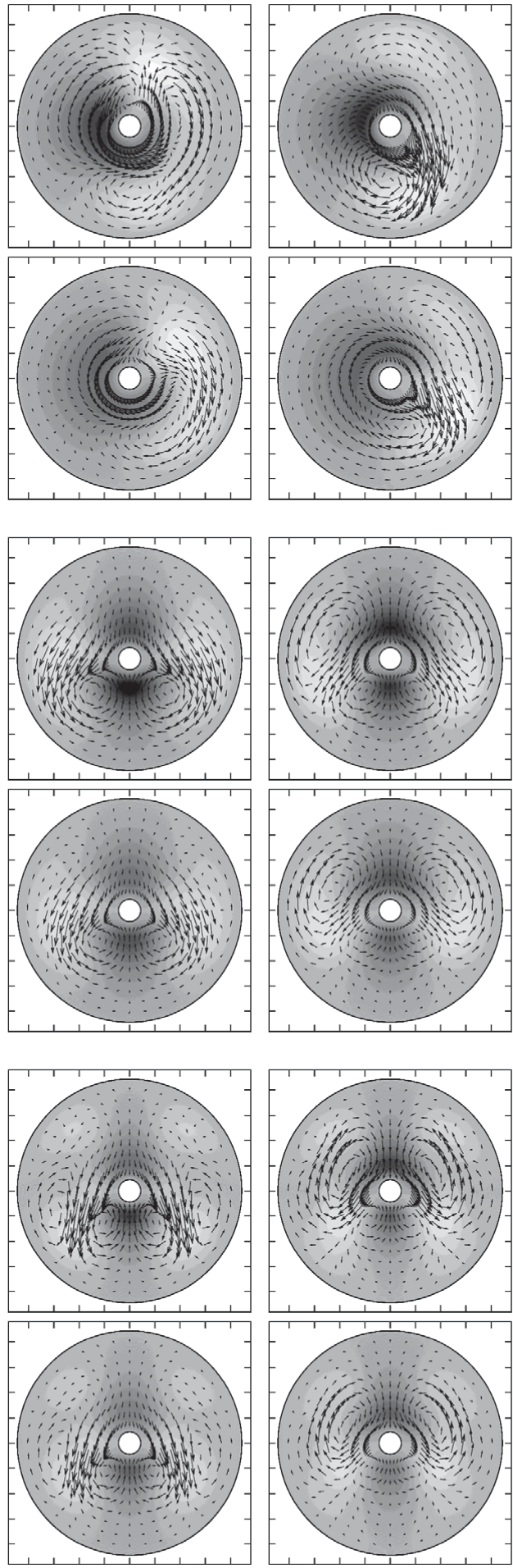

FiguRE 16. The disturbance flow field for $\left(\eta, m_{0}, R\right)=(0.1,1,300)$. The four parts of $(a)$ and (b) correspond to the upper and lower branch solutions respectively. The arrows indicate the projection of the velocity on the cross-section, $z=(k / 4)(2 \pi / \alpha), k=0,1,2,3$ (from left to right). Grey scale represents the axial velocity component (light : fast, dark : slow). ( $a$ ) The class $\mathscr{P}$ with $\alpha=0.59,(b)$ the class $\mathscr{M}$ with $\alpha=0.77$ and $(c)$ the class $\mathscr{M}$ with $\alpha=0.86$. 

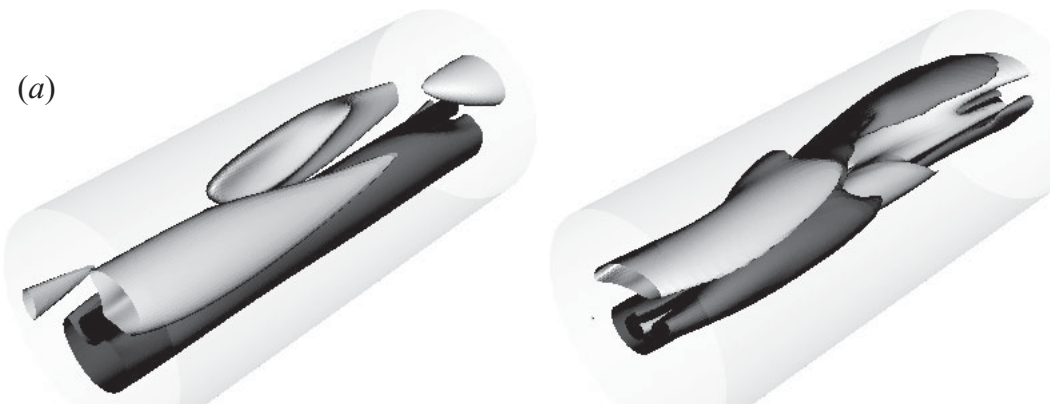

(b)
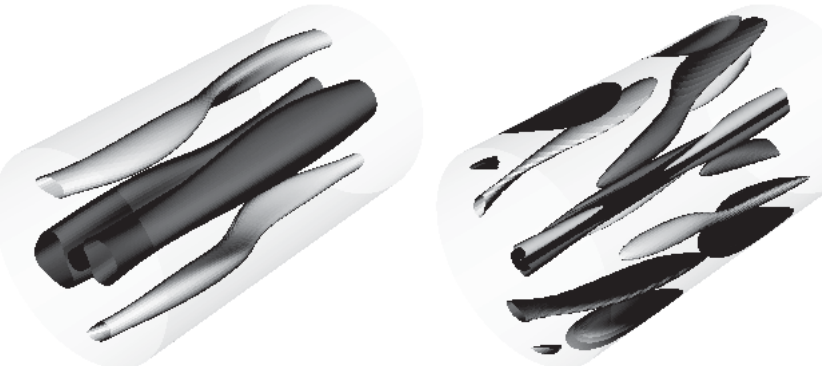

(c)
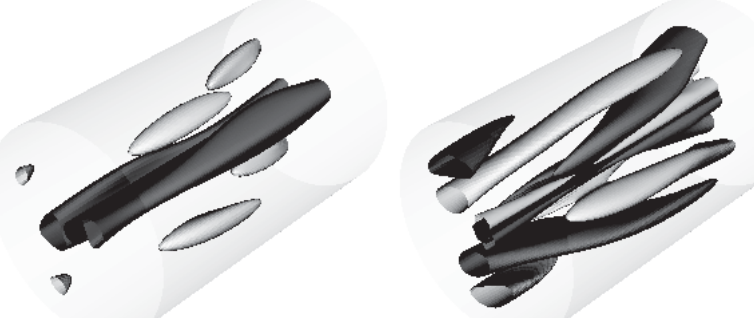

FIGURE 17. The axial variation of the streaks for the travelling wave for $\left(\eta, m_{0}, R\right)=$ $(0.1,1,300)$. Left, the isosurface of the axial velocity at $w-U_{B}= \pm 50$. The light/dark grey surfaces correspond to the fast/slow speed streaks. Right, the isosurface of the axial vorticity at $\omega_{z}= \pm 50$. The light/dark grey surfaces correspond to positive/negative vorticities. (a) The class $\mathscr{P}$ with $\alpha=0.59,(b)$ the class $\mathscr{M}$ with $\alpha=0.77$ and $(c)$ the class $\mathscr{M}$ with $\alpha=0.86$.

$$
\check{\check{w}}(x, t)=\sum_{l=0}^{\infty} \check{e}_{l} g_{l}(x) \exp (s t)
$$

where $c_{\theta}$ and $c_{z}$ are the phase velocities of the travelling wave solution. After truncating infinite series at the same level as the travelling wave solution, we obtain the linear algebraic eigenvalue problem. Using the same notation as (4.6), it becomes

$$
\left\{D_{i j}+H_{i j k} X_{k}+H_{i k j} X_{k}+\mathrm{i}\left(m m_{0} c_{\theta}+n \alpha c_{z}\right) B_{i j}\right\} \check{X}_{j}=s B_{i j} \check{X}_{j},
$$

where $\check{X}_{j} \in\left(\check{a}_{l m n}, \check{b}_{l m n}, \check{d}_{l}, \check{e}_{l}\right)$ and $X_{j} \in\left(a_{l m n}, b_{l m n}, d_{l}, e_{l}\right)$.

Our numerical analysis shows that (5.6) always has two zero eigenvalues. These eigenvalues result from the fact that SCF is invariant under azimuthal and axial translations. 

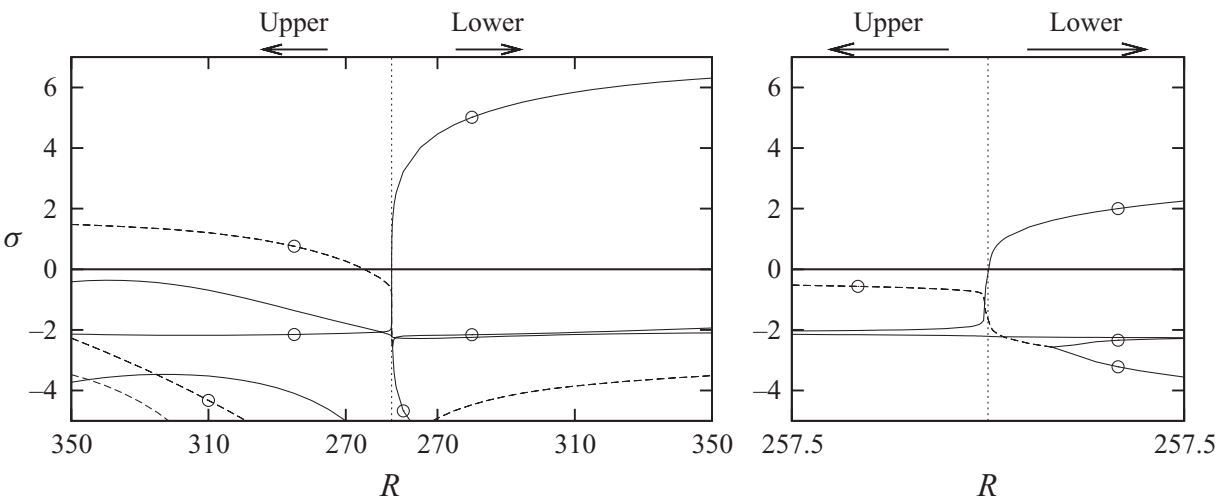

FIGURE 18. The real part $\sigma$ of the growth rate $s$ of the travelling wave solution for $\eta=0.5$ corresponding to those with $m_{0}=3$ at $\alpha=0.51$ in figure 9. The solid/dashed curves imply that the imaginary part $\gamma$ of the growth rate $s$ is zero/non-zero. The figure includes two growth rates $s \equiv 0$ for any $R$. The curves with an open circle correspond to the perturbation with symmetry (i). The right figure is a close-up near the saddle-node bifurcation point (dotted vertical line).

Figures 18 and 19 show the growth rate $s$ around the saddle-node in the case $\eta=0.5$ and 0.1 , respectively. Recall that the class $\mathscr{P}$ solution possesses symmetry (i) only, whereas the class $\mathscr{M}$ solution possesses symmetries (i) and (iii). The curves with an open circle in these figures correspond to the eigenvalues calculated by restricting $\check{X}_{j}$ to those with symmetry (i), and the curves with a solid circle are due to the restriction to $\check{X}_{j}$ with symmetry (iii). The curves with both open and solid circles correspond to the eigenvalues calculated by restricting $\check{X}_{j}$ to those with both symmetries (i) and (iii). It is seen that the lower branch solutions are always unstable against the fundamental mode and, consistent with the bifurcation theory, the real eigenvalue crosses zero at the saddle-node bifurcation point (dotted vertical line). It is found that the upper branch solutions are stable in a small region next to the saddle-node (except for figure $19(c)$ ). As $R$ increased, these stable solutions soon become unstable against the perturbation with symmetry (i): the corresponding eigenvalues are complex, and so some sorts of time-dependent solutions are expected.

\section{Conclusion}

The linear stability analysis of SCF with respect to axisymmetric perturbations by Gittler (1993) has been extended to the case with respect to arbitrary three-dimensional perturbations. We find that the findings of Gittler (1993), namely SCF becomes linearly unstable if $\eta<0.1415$, are still valid even for general three-dimensional stability analysis. The instability is determined by axisymmetric perturbations.

In our nonlinear analysis, we obtain finite-amplitude axisymmetric solutions travelling downstream. These solutions bifurcate subcritically from the linear critical points. The critical Reynolds numbers for $\eta<0.1415$ are extraordinarily large. In order to investigate the possibility for the existence of other types of nonlinear solutions in a relatively low Reynolds number region, we use the three-dimensional PCF equilibrium solution by Nagata (1990) in the limit of narrow gap $(\eta=1)$ and continue it to wider cases $(\eta<1)$. This homotopy approach proves to be successful and the resulting non-axisymmetric nonlinear solutions travelling in the axial direction are found. They are characterized by fast and slow speed streaks flanked by strong axial 
(a)

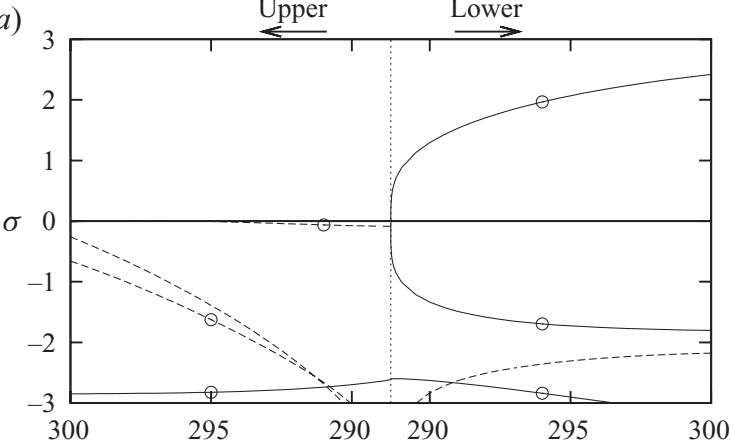

(b)

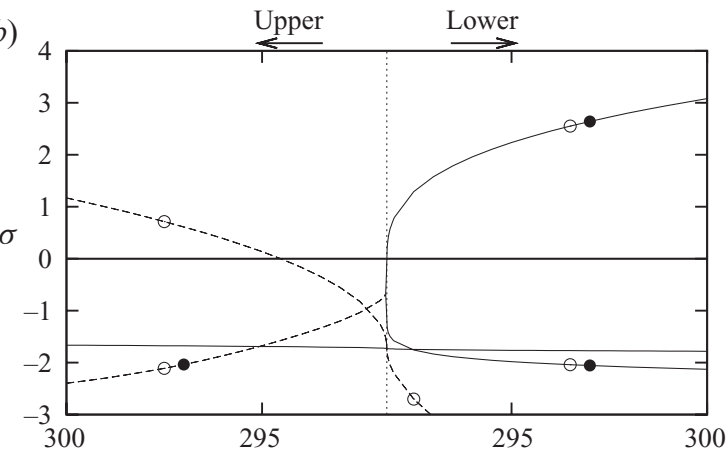

(c)

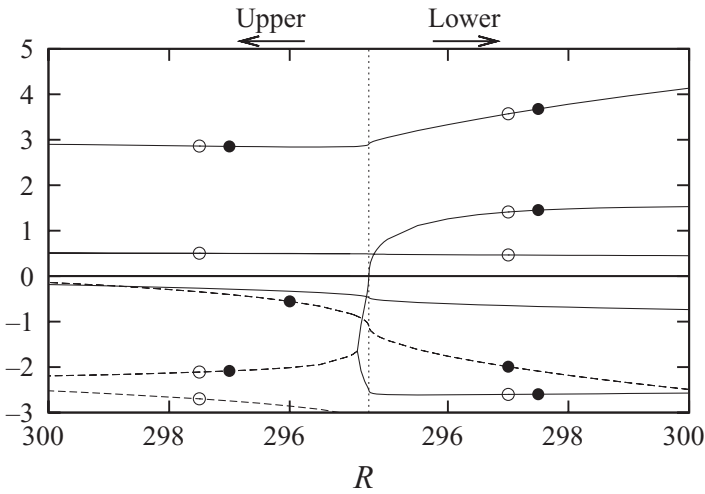

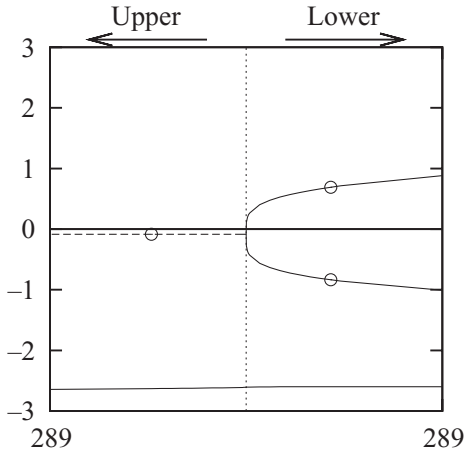

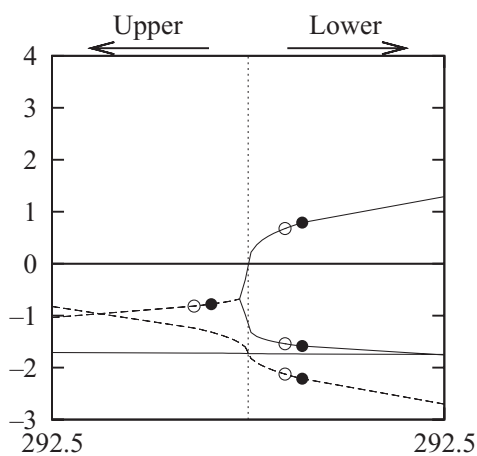
89

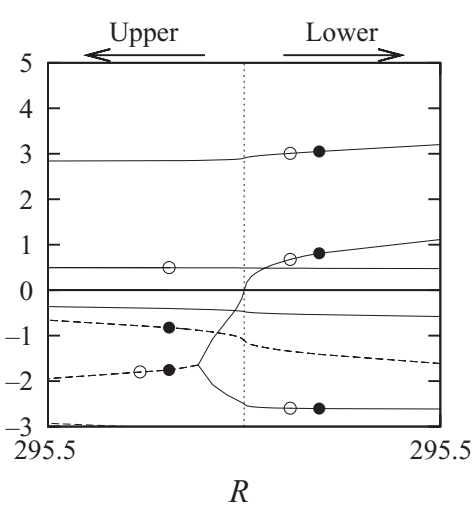

FIGURE 19. The real part $\sigma$ of the growth rate $s$ of the three travelling wave solutions $\left(\eta, m_{0}\right)=(0.1,1)$ corresponding to those in figure 15 . The solid/dashed curves imply that the imaginary part $\gamma$ of the growth rate $s$ is zero/non-zero. The figures include two growth rates $s \equiv 0$ for any $R$. The curves with an open or solid circle correspond to the perturbation with symmetry (i) or (iii), respectively. The curves with both open and solid circles correspond to the perturbation with both symmetries (i) and (iii). The righthand panel shows close-ups near the saddle-node bifurcation point (dotted vertical line). (a) The class $\mathscr{P}$ with $\alpha=0.59,(b)$ the class $\mathscr{M}$ with $\alpha=0.77$ and (c) the class $\mathscr{M}$ with $\alpha=0.86$.

vortices. These solutions result from saddle-node bifurcations. For $\eta=0.5$, where the linear critical state is absent, the Reynolds number at the saddle-node bifurcations reaches a minimum, approximately 256.6, when the azimuthal wavenumber $m_{0}=3$. We also obtain highly symmetric solutions for $\eta=0.1$. The linear stability analysis of non-axisymmetric travelling wave solutions, though restricted to the fundamental 


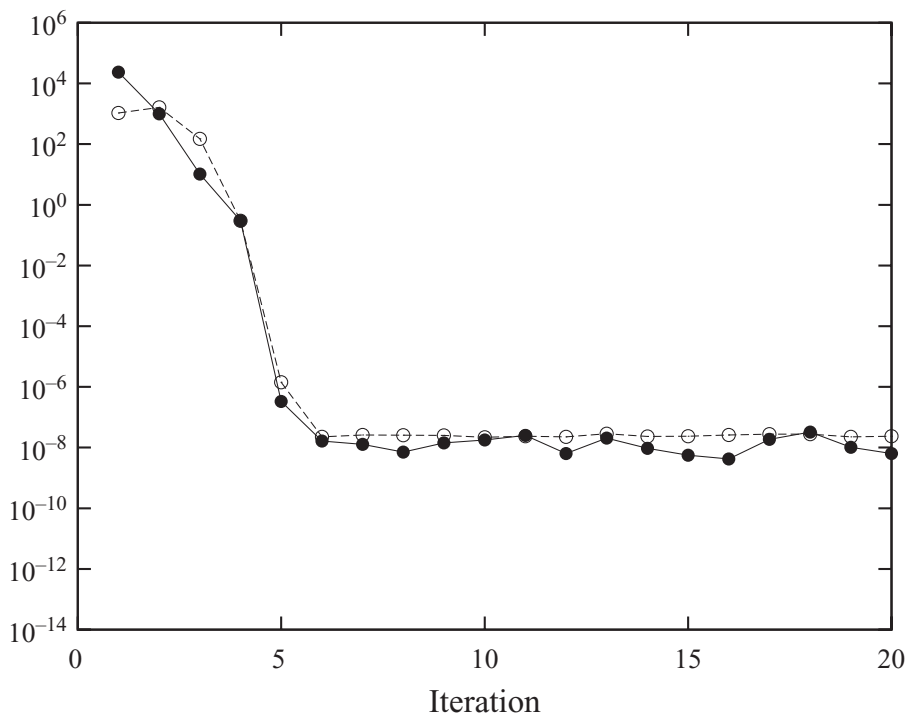

FIGURE 20. The accuracy of the solution for the nonlinear algebraic equation (4.5) based on the maximum relative error $\epsilon$ (solid circles) and the $L_{2}$-norm of the residual $\left\|F_{i}\right\|_{2}$ (open circles) as a function of the Newton-Raphson iteration. $\left(\eta, m_{0}, \alpha, R\right)=(0.5,3,0.50,350)$. $(L, M, N)=(16,8,9)$. Double precision is used.

mode, shows that the lower branch solutions are always unstable and that stable upper branch solutions may exist in a small region next to the saddle-node bifurcation point.

All of the present numerical investigations indicate subcritical transition to turbulence in SCF though the evidence for such a phenomenology remains to be seen.

The nonlinear travelling wave solutions obtained in the paper will be easily continued to the annular Couette-Poiseuille flow by adding an axial pressure gradient.

\section{Appendix. Convergence criterion of the Newton-Raphson method}

An example of the variation of $\epsilon$ in (4.6) as the Newton-Raphson iteration is repeated is shown in figure 20. The calculation is undertaken at $\left(\eta, m_{0}, \alpha, R\right)=$ $(0.5,3,0.50,350)$ with the resolution $(L, M, N)=(16,8,9)$. We use the converged solution at $\left(\eta, m_{0}, \alpha, R\right)=(0.5,3,0.51,350)$ as an initial guess. Double precision is used. We can see from the solid circles in figure 20 that the accuracy does not improve once the criterion (4.7) is satisfied at the fifth iteration. As for the $L_{2}$-norm of the residual of (4.5), $\left\|F_{i}\right\|_{2}$ is not reduced after the fifth iteration either (see the open circles in figure 20). However, the value of $\left\|F_{i}\right\|_{2}$ for the approximation obtained by using the criterion (4.6) is not so large as one would think, when it is compared with the typical order of the corresponding $\left\|L_{i}\right\|_{2}$ (see tables 2, 4 and 5). From figure 20 , the tolerance $10^{-5}$ in (4.6) guarantees convergence with the signal-to-noise-floor ratio at $O\left(10^{-11}\right)-O\left(10^{-12}\right)$.

\section{REFERENCES}

Arney, M. S., Bai, R., Guevara, E., Joseph, D. D. \& Liu, K. 1993 Friction factor and holdup studies for lubricated pipelining. I. Experiments and correlations. Intl J. Multiphase Flow 19, 1061-1076. 
Drazin, P. G. \& ReID, W. H. 1981 Hydrodynamic Stability. Cambridge University Press.

Faisst, H. \& Eckhardt, B. 2003 Traveling waves in pipe flow. Phys. Rev. Lett. 91, 224502.

Frei, Ch., Lüscher, P. \& Wintermantel, E. 2000 Thread-annular flow in vertical pipes. J. Fluid Mech. 410, 185-210.

Gibson, J. F., Halcrow, J. \& Cvitanovic, P. 2009 Equilibrium and travelling-wave solutions of plane Couette flow. J. Fluid Mech. 638, 243-266.

GitTler, P. 1993 Stability of Poiseuille-Couette flow between concentric cylinders. Acta Mechanica. 101, 1-13.

Joseph, D. D. 1976 Stability of Fluid Motions, Vols I and II. Springer.

MARQUES, F. 1990 On boundary conditions for velocity potentials in confined flows: application to Couette flow. Phys. Fluids A A2, 729-737.

Nagata, M. 1990 Three-dimensional finite amplitude solutions in plane Couette flow: bifurcation from infinity. J. Fluid Mech. 217, 519-527.

Okino, S., Nagata, M., Wedin, H. \& Bottaro, A. 2010 A new nonlinear vortex state in square duct flow. J. Fluid Mech. 657, 413-429.

Okino, S. \& Nagata, M. 2011 Asymmetric traveling waves in a square duct. J. Fluid Mech. (submitted).

Panoliaskos, A., Hallett, W. L. H. \& Garis, I. 1985 Prediction of optical fiber coating thickness. Appl. Opt. 24, 2309-2312.

Preziosi, L. \& Rosso, F. 1990 Stability of a viscous liquid between sliding pipes. Phys. Fluids A 2 (7), 1158-1162.

Pringle, C. C. T., Duguet, Y. \& Kerswell, R. R. 2009 Highly symmetric travelling waves in pipe flow. Phil. Trans. R. Soc. A 367, 457-472.

Pringle, C. C. T. \& Kerswell, R. R. 2007 Asymmetric, helical and mirror-symmetric traveling waves in pipe flow. Phys. Rev. Lett. 99 (2), 074502.

Sadeghi, V. M. \& Higgins, B. G. 1991 Stability of sliding Couette-Poiseuille flow in an annulus subject to axisymmetric and asymmetric disturbances. Phys. Fluids A 3 (9), 2092-2104.

Shands, J., Alfredsson, H. \& Lindgren, E. R. 1980 Annular pipe flow subject to axial motion of the inner boundary. Phys. Fluids 23 (10), 2144-2145.

TADMOR, Z. \& BIRD, R. B. 1974 Rheological analysis of stabilizing forces in wire-coating dies. Polym. Engng Sci. 14 (2), 124-136.

Uhlmann, M., Kawahara, G. \& Pinelli, A. 2010 Traveling-waves consistent with turbulence-driven secondary flow in a square duct. Phys. Fluids 22, 084102.

Vaskopulos, T., Polymeropoulos, C. E. \& Zebib, A. 1993 Heat transfer from optical fibre during the draw process. J. Mat. Proc. Manuf. Sci. 1, 261-271.

Walton, A. G. 2003 The nonlinear instability of thread-annular flow at high Reynolds number. J. Fluid Mech. 477, 227-257.

Walton, A. G. 2004 Stability of circular Poiseuille-Couette flow to axisymmetric disturbances. J. Fluid Mech. 500, 169-210.

Walton, A. G. 2005 The linear and nonlinear stability of thread-annular flow. Phil. Trans. R. Soc. A 363, 1223-1233.

WANG, J., Gibson, J. F. \& WALEFFE, F. 2007 Lower branch coherent states in shear flows: transition and control. Phys. Rev. Lett. 98, 204501.

Wedin, H., Bottaro, A. \& Nagata, M. 2009 Three-dimensional travelling waves in a square duct. Phys. Rev. E. 79, 065305.

Wedin, H. \& Kerswell, R. R. 2004 Exact coherent structures in pipe flow: travelling wave solutions. J. Fluid Mech. 508, 333-371. 